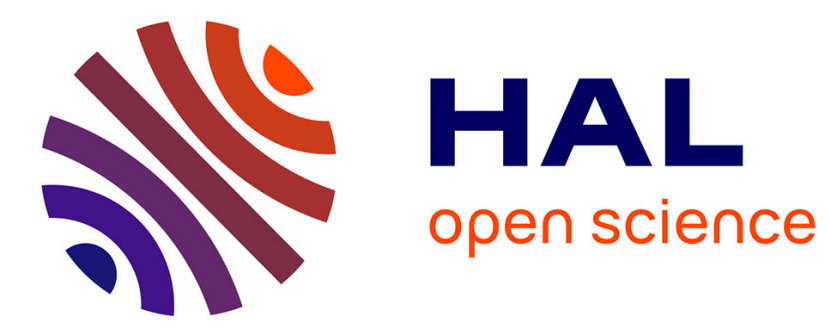

\title{
La représentation de points de vue dans le système d'aide à la décision en cancérologie KASIMIR
}

\author{
Mathieu d'Aquin, Jean Lieber, Amedeo Napoli
}

\section{To cite this version:}

Mathieu d'Aquin, Jean Lieber, Amedeo Napoli. La représentation de points de vue dans le système d'aide à la décision en cancérologie KASIMIR. Revue des Sciences et Technologies de l'Information Série L'Objet: logiciel, bases de données, réseaux, 2007, 13 (2-3), pp.143-175. inria-00201561

\section{HAL Id: inria-00201561 https://hal.inria.fr/inria-00201561}

Submitted on 31 Dec 2007

HAL is a multi-disciplinary open access archive for the deposit and dissemination of scientific research documents, whether they are published or not. The documents may come from teaching and research institutions in France or abroad, or from public or private research centers.
L'archive ouverte pluridisciplinaire HAL, est destinée au dépôt et à la diffusion de documents scientifiques de niveau recherche, publiés ou non, émanant des établissements d'enseignement et de recherche français ou étrangers, des laboratoires publics ou privés. 


\title{
La représentation de points de vue dans le système d'aide à la décision en cancérologie KASIMIR
}

\author{
Mathieu d'Aquin — Jean Lieber — Amedeo Napoli \\ LORIA - Equipe Orpailleur \\ Bâtiment B, BP 239, F-54506 Vandouvre les Nancy \\ $\{$ daquin, lieber, napoli\}@loria.fr
}

RÉSUMÉ. Dans cet article, nous décrivons la représentation de points de vue dans le système KASIMIR d'aide à la décision en cancérologie. Cette mise en æuvre théorique et pratique des points de vue se place dans la lignée des travaux sur le sujet en représentation des connaissances. Côté théorique, les points de vue sont considérés dans le cadre de la logique de descriptions distribuée $C$-OWL, qui autorise la représentation et la manipulation explicites de points de vue. Côté pratique, une implantation des points de vue (en C-OWL) dans une application pratique en cancérologie permet de détailler le raisonnement distribué entre les points de vue localement et globalement et montre qu'un nouveau pas est franchi dans la conception de représentation à points de vue multiples.

ABSTRACT. In this paper, we introduce the knowledge representation based on viewpoints on which relies the KASIMIR system, aimed at decision helping in oncology. The design of viewpoints is considered on both theoretical and practical levels, and takes its place in the range of work on the subject that has a rather long history in the domain of object-based knowledge representation systems. From the theoretical side, the viewpoints are considered within the distributed description logic C-OWL, that allows the explicit representation and manipulation of viewpoints. From the practical side, an operational implementation of viewpoints in $\mathrm{C}-\mathrm{OWL}$ within an application in oncology shows how viewpoints are designed, and how they can be used in local and global reasoning schemes across viewpoints.

MOTS-CLÉS : point de vue, contexte, ontologie, logique de descriptions, raisonnement multi point de vue, $C$-OWL, cancérologie.

KEYWORDS: viewpoint, context, ontology, description logics, viewpoint-based distributed reasoning, C-OWL, oncology.

DOI:10.3166/OBJ.13.2-3.143-175(c) 2007 Lavoisier, Paris

RSTI - L'objet - 13/2007. Points de vue et paradigmes proches, pages 143 à 175 


\section{Introduction}

Lorsqu' on parle du point de vue de quelqu'un dans le langage courant, il s'agit généralement de la position que prend cette personne concernant un sujet particulier. Ces deux termes - point de vue et position - relèvent d'ailleurs de la même image : celle du lieu où se trouve la personne vis-à-vis du sujet et ainsi de l'angle selon lequel elle le considère. Au-delà de cette vision très générale, des acceptions, nombreuses et différentes, de la notion de «point de vue» peuvent être exprimées, en particulier pour ce qui concerne la construction d'une ontologie. Dans ce cadre et de façon très générale, la représentation des connaissances consiste à modéliser et à formaliser les connaissances relatives à un domaine. Construire une base de connaissances ou une ontologie passe par le choix des objets, des concepts et des relations qu'il est pertinent de représenter, ainsi que par une sélection des caractéristiques des objets jugées utiles pour le traitement de l'application courante. En particulier, la construction d'une ontologie relève d'au moins trois acceptions différentes de la notion de point de vue : (i) une ontologie est une représentation particulière du monde, qui (ii) délimite des caractéristiques pertinentes pour les objets représentés et qui (iii) déclare ce qui doit être considéré comme vrai lors de son utilisation.

Dans cet article, nous nous intéressons à la représentation explicite de points de vue pour l'intégration et la coordination de plusieurs représentations alternatives des connaissances relatives à un domaine. Cet intérêt s'inscrit dans le cadre du projet KASIMIR, qui porte sur la représentation de connaissances pour l'aide à la décision en cancérologie. La cancérologie est un domaine complexe où différentes disciplines comme la chimiothérapie, la chirurgie, l'hormonothérapie et la radiothérapie, sont impliquées dans les différentes phases de la prise en charge d'un patient. Dans la plupart des cas, la décision à prendre peut être établie en fonction d'un référentiel, qui fait figure de protocole de décision dédié à une localisation cancéreuse particulière, comme par exemple le cancer du sein. Un référentiel synthétise les connaissances actuelles en médecine - selon les principes de la médecine factuelle ou Evidence Based Medicineet prend en compte les cas les plus courants. Il ne permet pas de fait de couvrir toutes les situations : pour les patients qui sortent du cadre du référentiel, pour cause de contre-indication par exemple, les décisions sont prises lors d'une réunion de concertation pluridisciplinaire (ou RCP). Ces réunions regroupent des experts des différentes disciplines de la cancérologie et ont pour objectif d'adapter les recommandations du référentiel aux cas particuliers pour lesquels le référentiel ne fournit pas de réponse satisfaisante. Lors de ces réunions, un expert de chaque discipline apporte sa part à la solution et collabore avec les autres experts pour établir une recommandation globale. Ainsi, chaque discipline apporte son point de vue sur la prise en charge du patient et s'appuie pour cela sur sa vision des connaissances du domaine, en relation avec les autres points de vue que constituent les autres disciplines.

En accord avec la façon dont se déroulent les choses dans la réalité, les connaissances dans le système KASIMIR sont structurées selon les différentes disciplines impliquées dans la prise en charge d'un patient : chimiothérapie, chirurgie, hormonothérapie et radiothérapie. Chaque discipline s'appuie sur une représentation particulière 
du domaine et considère les patients selon une optique propre : chaque discipline apporte son point de vue lors de la prise de décision et cette multiplicité des points de vue doit (obligatoirement) être prise en compte dans la représentation globale des connaissances.

La mise en œuvre théorique et pratique des points de vue dans le système KASIMIR se place dans la lignée des travaux sur le sujet en représentation des connaissances, sujet que cet article entend réactiver d'ailleurs, dans le contexte général du web sémantique. Côté théorique, les points de vue sont considérés dans le cadre du formalisme C-OWL - C-OWL est une logique de descriptions distribuée, extension de OWL, le langage de représentation d'ontologies du web sémantique (Fensel et al., 2003; Staab et al., 2004) - qui autorise la représentation et la manipulation explicites de points de vue. Côté pratique, une implantation des points de vue en C-OWL dans une application en cancérologie permet de détailler le raisonnement distribué entre les points de vue localement et globalement. Cet article reprend et actualise les grandes lignes d'un premier travail des auteurs sur les points de vue dans une représentation par objets (d'Aquin et al., 2004b). Ici, c'est la présentation d'un travail plus accompli qui se place dans le cadre différent et mieux approprié de la logique de descriptions distribuée C-OWL qui est proposée.

C-OWL est particulièrement bien adapté à la problématique de la représentation de points de vue multiples, au moins dans le cadre du projet KASIMIR. Les disciplines impliquées dans la prise en charge des patients atteints de cancers correspondent à autant de sous-domaines de la cancérologie. Chaque discipline possède une organisation propre des connaissances qui la concernent et ne considère pas les mêmes éléments comme pertinents ou au premier plan. Il devient alors naturel d'associer à ces différentes disciplines différentes ontologies locales - des contextes en C-OWL - et de modéliser la collaboration et le partage de connaissances par des passerelles entre les ontologies locales.

Un nouveau pas est sans aucun doute franchi ici dans la représentation de points de vue en représentation de connaissances par objets et dans les logiques de descriptions. En effet, et il est important de le souligner, le travail de recherche sur l'introduction de points de vue dans le système KASIMIR a donné lieu à un système opérationnel, qui, à notre connaissance, est le seul traitant ce type d'application et mettant en jeu la logique de descriptions distribuée C-OWL. Le résultat est tangible et l'existence et la fonction même des points de vue dans le système KASIMIR fournissent une preuve pratique aux développeurs de C-OWL du bien-fondé de leur travail de recherche pratique et théorique sur les logiques de descriptions distribuées. En parallèle, un autre des objectifs pratiques du projet KASIMIR est la conception d'un portail sémantique dédié à la gestion des connaissances en cancérologie (d'Aquin et al., 2005). En tant qu'extension de OWL, le formalisme C-OWL et les mécanismes de raisonnements afférents s'intègrent naturellement dans l'infrastructure du web sémantique et donc dans l'environnement du portail sémantique de KASIMIR.

L'article est articulé comme suit. De nombreux systèmes, en particulier en représentation des connaissances par objets, ont intégré la notion de point de vue, sous des 
formes différentes et variées. Nous nous attachons dans un premier temps à donner un aperçu de ces systèmes et des langages de représentation multi point de vue qu'ils implémentent. Nous abordons aussi une notion proche de celle de points de vue, qui est celle de contexte, telle qu'elle est considérée en représentation des connaissances. Nous montrons ensuite comment les points de vue s'intègrent à la notion d'ontologie, notion essentielle dans le domaine du web sémantique. En nous appuyant sur le langage C-OWL - pour contextualized web ontology language, les points de vue étant considérés ici comme des contextes - nous introduisons et détaillons une démarche pour la modélisation et la représentation d'ontologies à points de vue multiples. Cette démarche est mise en œuvre dans le cadre du projet KASIMIR pour la représentation de connaissances décisionnelles en cancérologie, proposant ainsi une application à l'échelle du monde réel de la représentation de connaissances et du raisonnement avec points de vue multiples. Une discussion qui reprend les différents points forts relatifs à l'introduction de points de vue et à l'utilisation d'un formalisme de représentation comme C-OWL et une conclusion intégrant les perspectives de ce travail de recherche terminent cet article.

\section{Les points de vue en représentation des connaissances}

Il existe généralement plusieurs façons d'appréhender les éléments de connaissances relatifs à un domaine, qui peuvent être autant de différents points de vue selon lesquels représenter ces connaissances. Par exemple, en fonction de la tâche à accomplir, une voiture sera considérée de différentes façons ou points de vue : l'intérêt pourra porter sur sa consommation et sur son prix d'achat selon le point de vue économique, sur le nombre de places et la taille du coffre si c'est l'aspect fonctionnel qui l'emporte, ou encore sur la couleur et la forme s'il est plus opportun de se focaliser sur l'esthétique de la voiture. Les travaux en représentation des connaissances se sont ainsi souvent intéressés à faire coexister plusieurs représentations alternatives dans une même base de connaissances, en intégrant de façon explicite la notion de point de vue.

Cette section donne un aperçu des grandes tendances dans les systèmes et les langages de représentation à points de vue multiples. Les travaux portant sur les points de vue et plus en rapport avec la programmation par objets ou les bases de données ne sont pas abordés ici, sauf s'ils présentent un lien direct avec la représentation des connaissances.

\subsection{Les points de vue en représentation des connaissances par objets}

C'est en représentation des connaissances par objets (RCO (Masini et al., 1989; Ducournau, 1998; Euzenat, 1998)) qu'un grand nombre de systèmes de représentation de connaissances multi point de vue a été développé. La représentation des connaissances par objets repose généralement sur la notion de classe pour représenter des ensembles d'objets. Les classes se voient associer des attributs et sont organisées en 
une hiérarchie. Une classe dans cette hiérarchie hérite les attributs de toutes ses superclasses. Certains systèmes autorisent qu'une classe ait plusieurs superclasses directes (héritage multiple). Un objet est instance d'une ou de plusieurs classes et hérite les attributs de cette ou de ces classes.

KRL (Knowledge representation language (Bobrow et al., 1977)) est sûrement le premier système de RCO permettant la représentation de points de vue (appelés perspectives en KRL). Comme dans la plupart des systèmes ultérieurs, la notion de point de vue est intégrée en permettant qu'un objet soit associé à plusieurs représentations différenciées, à travers plusieurs classes. Les objets de KRL (individuals) peuvent ainsi appartenir à plusieurs classes, chacune regroupant les attributs pertinents selon un point de vue particulier. En ce sens, la représentation multi point de vue dans ce langage relève principalement de l'instanciation multiple. Il est possible de décrire en KRL une expression traduisant la phrase suivante :

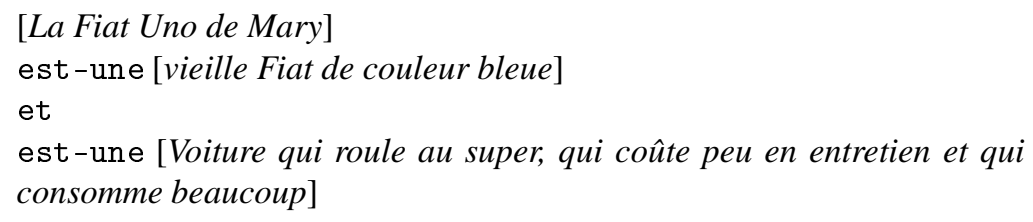

pour représenter deux points de vue différents - esthétique et économique - sur l'objet La Fiat Uno de Mary.

Dans le système Troeps (Mariño et al., 1990; Mariño, 1993), un concept représente, au même titre qu'une classe, un ensemble d'objets. Chaque concept se voit associer un ensemble de points de vue et un point de vue correspond à une hiérarchie de classes au sens de la RCO classique. Ainsi, TROEPS ne considère pas, comme il est habituel de le faire dans les RCO et les logiques de descriptions, les termes «classe » et « concept » comme des synonymes. Par principe, une hiérarchie de classes en TROEPS est à héritage simple, où chaque classe n'a qu'un seul ascendant direct. Les objets en TROEPS sont instances d'un concept et toute instance d'un concept doit nécessairement être instance d'une classe unique dans chacun des points de vue du concept. Par exemple, la figure 1 montre la représentation du concept de voiture, avec les trois points de vue esthétique, économique et fonctionnel. Le point de vue esthétique met en jeu des attributs comme le modèle, la taille ou la couleur de la voiture, le point de vue économique porte sur le prix d'achat et la consommation en carburant par exemple, et enfin le point de vue fonctionnel porte plutôt sur le nombre de places et la taille du coffre. La Fiat Uno de Mary est ainsi décrite comme une instance du concept de voiture et représentée par une classe de chacun des trois points de vue sur les voitures.

TROEPS permet de définir des liens, appelés passerelles, entre les points de vue. Une passerelle est une relation entre deux classes de deux points de vue différents d'un même concept. Elle est définie par une «classe d'origine » et une «classe de destination», pour indiquer que toute instance de la classe d'origine est nécessairement instance de la classe de destination. En ce sens, une passerelle s'interprète comme 


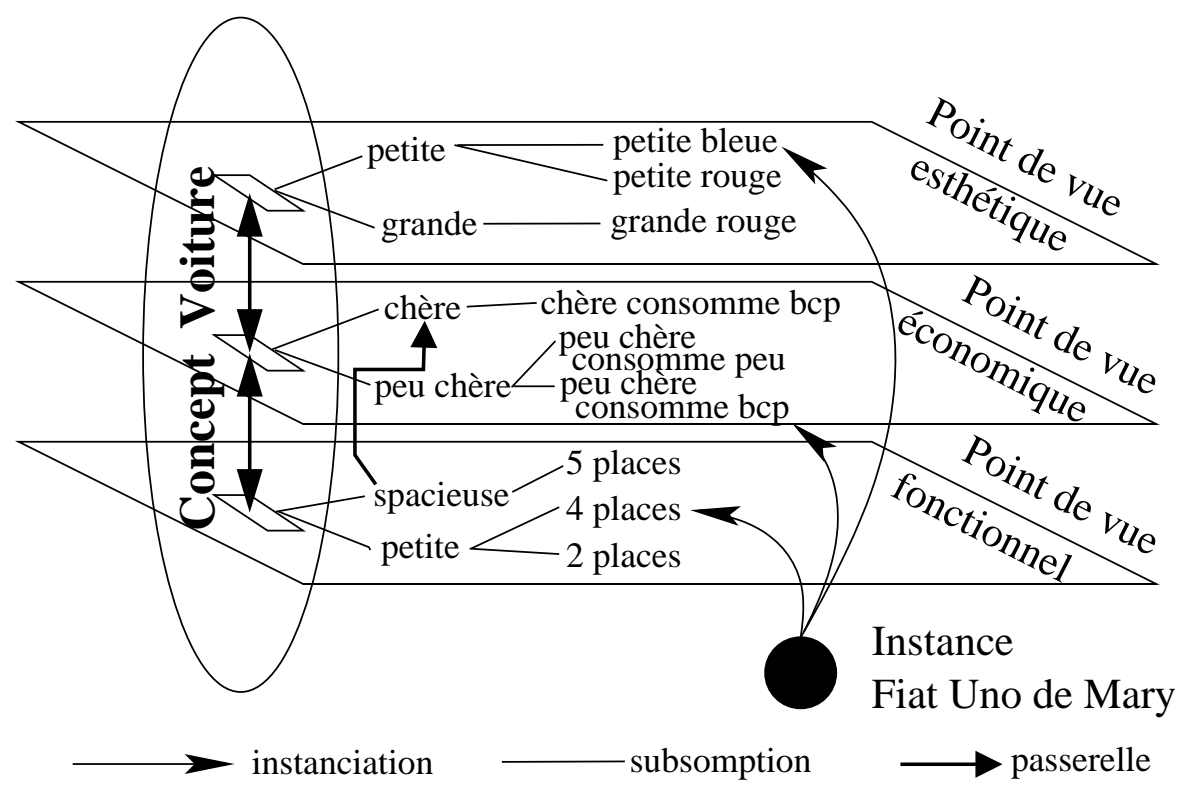

Figure 1. Un exemple de représentation du concept Voiture avec trois points de vue en TROEPS

une implication. Elle se rapproche par ailleurs de la relation de coréférence définie dans (Ferber et al., 1988; Ferber, 1989). Par exemple, on peut spécifier à l'aide d'une passerelle que les voitures spacieuses du point de vue fonctionnel sont chères du point de vue économique (voir figure 1). Des passerelles sont implicitement déclarées entre les classes racines des hiérarchies qui constituent les points de vue d'un concept. Ces passerelles matérialisent le fait que toute instance d'un concept est instance d'une classe de chacun des points de vue du concept.

Dans le cadre de la représentation à points de vue multiples, les systèmes ROME (Carré, 1989; Carré et al., 1990) et Frome (Dekker, 1994) distinguent deux relations différentes pour associer un objet à une classe : le lien d'instanciation et le lien de représentation. Le lien d'instanciation relie l'objet à la classe dont il est instance (à la façon des langages de programmation par objets). Le lien de représentation associe l'objet aux classes qui le représentent dans différents points de vue. De plus, il existe une relation d'exclusion mutuelle entre classes qui permet de spécifier que deux classes ne peuvent représenter un même objet, c'est-à-dire qu'elles déterminent deux points de vue incompatibles pour cet objet.

La figure 2 montre un exemple de représentation multi point de vue avec FrOME, qui peut se comparer avec la même représentation en KRL et en TROEPS. 


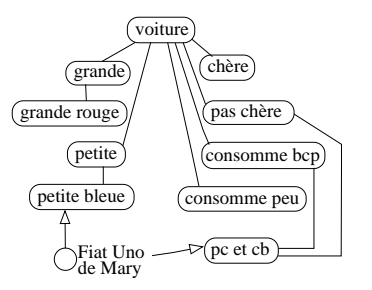

$K R L$

- subsomption

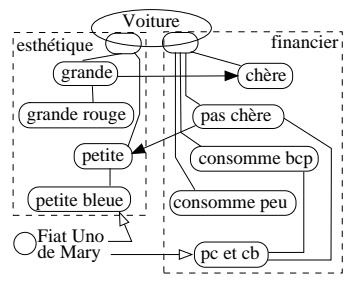

TROEPS

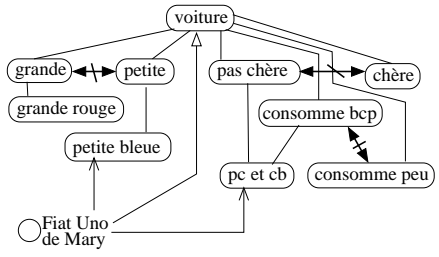

FROME

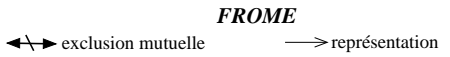

Figure 2. Points de vue en RCO

\subsection{Les points de vue en logique}

L'étude présentée dans (Hautamäki, 1986) vise à définir la notion de point de vue dans un cadre philosophique et logique. Cette étude montre l'importance de la notion de point de vue pour la représentation des connaissances et met l'accent sur l'aspect «ontologique» de la notion de point de vue «lorsqu'il s'agit de représenter les caractéristiques d'un être ». L'auteur part de l'idée qu'un point de vue correspond à une vue partielle sur un objet, c'est-à-dire à une représentation incomplète des informations le concernant. Il définit alors la syntaxe et la sémantique d'une logique permettant la manipulation de points de vue en tant que sélections dans les caractéristiques des objets.

(Attardi et al., 1995) suivent l'idée que différents points de vue expriment différentes visions relatives de la « vérité », au sens logique du terme. Ils formalisent cette notion dans une extension de la logique des prédicats. Un point de vue est alors défini comme un ensemble d'assertions de la logique et assimilé à une « microthéorie». L'extension comprend un métalangage et un ensemble de règles d'inférence permettant de raisonner dans et sur les points de vue. Un point de vue s'exprime dans le métalangage à l'aide de prédicats de la forme $i n\left({ }^{\prime} A^{\prime}, p d v\right)$, ce qui signifie intuitivement que « $A$ est vraie dans le point de vue $p d v$ », où $A$ est une formule de la logique et $p d v$ un symbole dénotant un point de vue. De plus, la formule $A$ dans in $\left({ }^{\prime} A^{\prime}, p d v\right)$ pouvant elle-même contenir des prédicats $i n$, il est possible dans un point de vue de raisonner sur le contenu d'autres points de vue. Par exemple, la propriété dite d'introspection positive s'exprime dans ce cadre par :

$$
i n\left({ }^{\prime} A^{\prime}, v p\right) \Longrightarrow i n\left({ }^{\prime} i n\left({ }^{\prime} A^{\prime}, v p\right)^{\prime}, v p\right)
$$

Représenter les points de vue par des ensembles d'assertions permet d'en faciliter la manipulation. Grâce à cela, il est possible en effet de définir très simplement des opérateurs comme l'union et l'intersection de points de vue, ainsi qu'une relation de subsomption - dénotée par $\succcurlyeq-$ permettant de hiérarchiser les points de vue : 


$$
p d v 2 \succcurlyeq p d v 1 \quad \Leftrightarrow \quad \forall A, i n\left({ }^{\prime} A^{\prime}, p d v 1\right) \Rightarrow i n\left({ }^{\prime} A^{\prime}, p d v 2\right)
$$

\subsection{Les points de vue et les graphes}

Dans (Ribière et al., 1997), une extension du formalisme des graphes conceptuels (GC) pour la représentation explicite de points de vue est présentée. Une correspondance élémentaire et partielle peut être établie entre GC et RCO. Ce qui est appelé en $\mathrm{RCO}$ un objet correspond à un individu en GC et les classes correspondent aux types de concepts. En GC, les concepts forment les nœuds de graphes dont les arcs sont des relations, qui jouent ainsi le rôle des attributs de la RCO. Les types de concepts sont organisés en une hiérarchie (un treillis de types), un type de concept étant considéré comme un sous-type d'un type de concept plus général. Les points de vue sont intégrés au formalisme des GC en donnant une «étiquette» dépendant d'un point de vue à la relation de sous-typage entre deux types de concepts. Par exemple, il peut être déclaré par ce biais que VoitureChère est un sous-type de Voiture selon le point de vue économique et que VoitureSpacieuse est un sous-type de Voiture selon le point de vue fonctionnel. En ce sens, la représentation de points de vue dans ce formalisme est proche de celle du système TROEPS (dont (Ribière et al., 1997) s'inspire d'ailleurs explicitement). De plus, des relations d'inclusion et d'exclusion peuvent être déclarées entre types de concepts, jouant les mêmes rôles que les passerelles de Troeps et les relations d'exclusions mutuelles de Frome.

Le système VIEWS (Davis, 1987) permet de représenter des objets structurés sous la forme de vues, où une vue est un réseau de parties (les nœuds du réseau), de relations et de contraintes. La représentation multi point de vue, dite aussi «multi perspective», de VIEWS donne la possibilité de décrire plusieurs vues contenant des éléments communs (identifiés par leurs noms). En outre, il est possible d'établir des relations entre les différentes vues.

\subsection{Les points de vue et les contextes}

La notion de contexte, telle qu'elle est considérée en représentation des connaissances, est très proche de la notion de point de vue. En effet, (Benerecetti et al., 2001) indiquent que dans une représentation multicontexte, deux contextes se distinguent soit parce qu'ils couvrent différentes «parties» du domaine, soit parce qu'ils se placent à différents niveaux d'approximation, ou encore parce qu'ils considèrent le monde selon différentes perspectives. Ainsi, il semble que la notion de contexte soit plus générale que celle de point de vue, et donc, qu'un modèle de représentation multicontexte doit pouvoir être utilisé pour représenter des points de vue multiples.

Une référence toujours actuelle en ce qui concerne la représentation et le raisonnement avec des contextes est incontestablement la formalisation proposée dans (McCarthy, 1993). Dans cette formalisation, toute assertion $A$ de la logique doit né- 
cessairement être réalisée dans un contexte, ce qui est dénoté par une expression de la forme $c: A$, où $c$ fait référence au contexte. Les contextes s'expriment par ailleurs par l'intermédiaire d'un prédicat $i s t$, pour is true, dans des expressions de la forme $c^{\prime}$ : ist $(c, p)$ signifiant intuitivement que la proposition $p$ est considérée comme vraie dans le contexte $c$, l'assertion étant elle-même donnée dans le contexte $c^{\prime}$. Dans cette formule, $c^{\prime}$ est considéré comme un contexte englobant pour $c$. Dans un contexte $c^{\prime \prime}$ englobant $c^{\prime}$, la proposition $i s t\left(c^{\prime}, i s t(c, p)\right)$ peut alors être vérifiée.

Les systèmes à contextes multiples (ou multicontextes, ce qui correspond à l'anglais, MCS pour multi-context systems) ont été présentés en premier lieu dans (Guinchiglia et al., 1994) comme une alternative aux logiques modales. En effet, les points de vue et les contextes semblent entretenir des liens étroits avec les logiques modales. (Attardi et al., 1995) montrent par exemple comment la formalisation des points de vue introduite permet la définition de modalités telles que la croyance et la connaissance. Les systèmes à contextes multiples s'appuient sur un cadre logique, où sont définies des règles d'inférence autorisant le raisonnement en fonction de contextes multiples. Ces travaux ont été par la suite complétés dans (Ghidini et al., 2001) par la définition d'une sémantique «à modèles locaux » (en anglais, LMS, local model semantics), proposant une formalisation de la notion de contexte dans le cadre de la théorie des modèles (model-theoric semantics). En LMS/MCS, un contexte est considéré comme une théorie partielle et approximative du monde, traduisant une perspective particulière. Les raisonnements sont principalement réalisés localement, au sein des contextes, tandis que les contextes sont reliés par des passerelles, ce qui permet la collaboration de raisonnements locaux.

Le formalisme C-OWL, qui est conçu pour représenter la notion de contexte dans les ontologies du web sémantique, s'appuie sur ces travaux de recherche. Ce formalisme se présente comme une extension de OWL pour la représentation d'ontologies contextuelles ou contexte (Bouquet et al., 2004). Un contexte en C-OWL est considéré comme une ontologie locale, représentée en OWL, possédant son propre langage et sa propre interprétation. Les éléments de chaque contexte, ce qui comprend les classes, les propriétés et les individus, peuvent être reliés entre eux par des passerelles. Par exemple, une passerelle de la forme $\mathrm{i}: \mathrm{C} \stackrel{\sqsubseteq}{\longrightarrow} \mathrm{j}: \mathrm{D}$ indique que la classe $\mathrm{C}$ telle qu'elle est représentée dans le contexte $O_{i}$ est vue par le contexte $O_{j}$ comme étant plus spécifique que la classe $\mathrm{D}$. Ces passerelles ont une sémantique bien définie qui permet de réaliser des raisonnements globaux, en réutilisant les connaissances d'un contexte à l'autre. Les fonctionnalités en rapport avec ce qui nous préoccupe ici, à savoir la représentation explicite et opérationnelle de points de vue, sont expliquées en détail dans la section 3.

Reste une autre question : est-ce que ces contextes sont seulement des points de vue, ou encore, quel est le rapport plus exact existant entre les contextes de la théorie LMS/MCS, les points de vue en représentation des connaissances selon TROEPS par exemple, et les contextes dans toute leur généralité ? La réponse à cette question complexe n'est pas simple : pour notre part, nous montrons ci-après comment nous avons adapté la notion de contexte en C-OWL pour représenter des points de vue 
en représentation des connaissances (dans un idiome proche de celui de TROEPS). D'une certaine façon, notre objectif est atteint mais la question reste ouverte : nous avons montré que les contextes de la théorie LMS/MCS, tels qu'il est possible de les implanter avec C-OWL, permettent une représentation relativement fidèle et surtout opérationnelle en logique de descriptions de la notion de point de vue.

\section{C-OWL et les ontologies contextuelles}

C-OWL est une extension de OWL pour la représentation d'ontologies contextuelles ou contextualisées (Bouquet et al., 2004). Les ontologies contextuelles sont des représentations locales, appelées contextes, qui sont en relation avec d'autres contextes par l'intermédiaire d'appariements. C-OWL a été conçu à l'origine avec l'objectif de servir de base à l'alignement d'ontologies (Shvaiko et al., 2005). Il est montré dans la suite comment C-OWL peut servir de cadre à la représentation de points de vue multiples sur les connaissances d'un domaine donné.

Afin de bien comprendre les principes sur lesquels repose le formalisme C-OWL, un bref rappel des fonctionnalités de OWL est tout d'abord proposé, suivi par une présentation détaillée et méthodologique de C-OWL.

\subsection{Une brève introduction à $O W L$}

OWL est le formalisme standard choisi pour la représentation d'ontologies pour le web sémantique. Une ontologie en OWL contient les définitions des classes, des relations ou propriétés et des individus du domaine concerné. Une classe représente un ensemble d'individus ou objets du domaine. Une propriété dénote une relation binaire entre les objets.

Formellement et à l'image des logiques de descriptions (Baader et al., 2003), la sémantique d'une ontologie en OWL s'exprime à l'aide d'une interprétation $\mathcal{I}=$ $\left(\Delta^{\mathcal{I}},{ }^{\mathcal{I}}\right)$, où $\Delta^{\mathcal{I}}$ est un ensemble non vide appelé domaine d'interprétation et où ${ }^{\mathcal{I}}$ est une fonction d'interprétation. Cette fonction associe à une classe $\mathrm{C}$ un sous-ensemble $\mathrm{C}^{\mathcal{I}}$ du domaine d'interprétation $\Delta^{\mathcal{I}}$, à une propriété $\mathrm{p}$ un sous-ensemble $\mathrm{p}^{\mathcal{I}}$ du produit cartésien $\Delta^{\mathcal{I}} \times \Delta^{\mathcal{I}}$ et à un individu a un élément a ${ }^{\mathcal{I}}$ de $\Delta^{\mathcal{I}}$. Pour des raisons de lisibilité et de simplicité, la syntaxe des logiques de descriptions est utilisée dans la suite de cet article pour décrire les expressions de OWL et C-OWL.

La syntaxe de OWL comprend un ensemble de constructeurs qui permettent de décrire le contenu des classes, comme la conjonction de classes, la quantification universelle et existentielle, la cardinalité des propriétés, etc. En particulier, la conjonction se note $C \sqcap D$ et s'interprète comme l'intersection $C^{\mathcal{I}} \cap D^{\mathcal{I}}$, la quantification existentielle se note $\exists \mathrm{p} . C$ et s'interprète comme l'ensemble $(\exists \mathrm{p} . \mathrm{C})^{\mathcal{I}}$ des objets en relation par l'intermédiaire de $\mathrm{p}$ avec au moins un élément de $\mathrm{C}^{\mathcal{I}}$. La syntaxe et la sémantique de l'ensemble global des constructeurs de OWL sont décrites dans (WOWG, 2004; Antoniou et al., 2004; Staab et al., 2004). 
En OWL, les classes sont introduites par des axiomes de la forme $\mathrm{C} \sqsubseteq \mathrm{D}$, où $\mathrm{C}$ et $\mathrm{D}$ sont des classes et où $\sqsubseteq$ dénote la relation de subsomption entre les classes. Formellement, une interprétation $\mathcal{I}$ satisfait $\mathrm{C} \sqsubseteq \mathrm{D}$ si et seulement si $\mathrm{C}^{\mathcal{I}} \subseteq \mathrm{D}^{\mathcal{I}}$. la notation $\mathrm{C} \equiv \mathrm{D}$ est une abréviation pour $\mathrm{C} \sqsubseteq \mathrm{D}$ et $\mathrm{D} \sqsubseteq \mathrm{C}$. Les assertions sont utilisées pour introduire les individus et ont deux formes possibles : $C(a)$ et $p(a, b)$, où $C$ est une classe, $a$ et $b$ sont deux individus et $\mathrm{p}$ est une propriété. L'interprétation $\mathcal{I}$ satisfait $\mathrm{C}$ (a) si et seulement si $\mathrm{a}^{\mathcal{I}} \in \mathrm{C}^{\mathcal{I}} ; \mathcal{I}$ satisfait $\mathrm{p}(\mathrm{a}, \mathrm{b})$ si et seulement $\mathrm{si}\left(\mathrm{a}^{\mathcal{I}}, \mathrm{b}^{\mathcal{I}}\right) \in \mathrm{p}^{\mathcal{I}}$.

Une ontologie $O$ en OWL est un ensemble d'axiomes et d'assertions. L'interprétation $\mathcal{I}$ est un modèle pour $O$ si $\mathcal{I}$ satisfait tous les axiomes et assertions de $O$.

\subsection{La syntaxe et la sémantique de C-OWL}

En C-OWL, les connaissances sont exprimées par l'intermédiaire d'un ensemble de contextes, appelé l'espace des contextes. Chaque contexte $O_{i}$ de cet espace est une ontologie en OWL, possédant son propre langage et sa propre interprétation. Formellement, un espace de contextes contient un ensemble de contextes $\left\{O_{i}\right\}_{i \in I}, I$ étant l'ensemble des index des contextes. Les index de $I$ sont utilisés pour préfixer les expressions en OWL dans le contexte courant, associant ainsi chaque expression au contexte auquel elle appartient. Par exemple, i: C, i: $\exists$ p. C, i:a, i:C $\sqsubseteq$ D et i:C (a) sont des exemples d'expressions déclarées dans le contexte $O_{i}$. La sémantique d'un espace de contextes s'exprime à l'aide d'une interprétation distribuée I, comprenant une interprétation locale $\mathcal{I}_{i}$ pour chaque $i \in I$. De façon classique, chaque $\mathcal{I}_{i}$ se définit par un domaine d'interprétation local $\Delta^{\mathcal{I}_{i}}$ et par une fonction d'interprétation locale $\cdot{ }^{\mathcal{I}_{i}}$. Un contexte est interprété en termes de l'interprétation locale correspondante, c'est-à-dire que $\mathfrak{I}$ satisfait un axiome ou une assertion dans $O_{i}$ si et seulement si $\mathcal{I}_{i}$ satisfait cet axiome ou cette assertion.

Les appariements ou mises en correspondance entre les contextes sont donnés par des passerelles : une passerelle entre les contextes $O_{i}$ et $O_{j}$ permet de déclarer une correspondance entre des éléments de connaissances existants dans chacun de ces deux contextes. Sur la base de ces correspondances, une partie des connaissances contenues dans $O_{i}$ peut être interprétée et réutilisée dans $O_{j}$.

Plus formellement, un appariement $\mathcal{M}_{i j}$ est un ensemble de passerelles de $O_{i}$ vers $O_{j}$, qui sont déclarées entre classes, propriétés ou individus, et qui possèdent des types différents. En particulier, une passerelle en-dessous est de la forme $\mathrm{i}: \mathrm{C} \stackrel{\sqsubseteq}{\longrightarrow}: \mathrm{D}$, où $i: \mathrm{C}$ et $\mathrm{j}$ :D sont des classes respectivement dans $O_{i}$ et $O_{j}$ et signifie que la classe $i: \mathrm{C}$ de $O_{i}$ est considérée, du point de vue de $O_{j}$, comme plus spécifique que la classe j :D (Serafini et al., 2005).

La passerelle au-dessus $\mathrm{i}: \mathrm{C} \stackrel{\sqsupseteq}{\longrightarrow} \mathrm{j}: \mathrm{D}$ signifie au contraire que $O_{j}$ considère la classe $i: \mathrm{C}$ comme plus générale que $\mathrm{j}: \mathrm{D}$. Ainsi, les passerelles sont directionnelles : une passerelle de $O_{i}$ vers $O_{j}$ est considérée du point de vue de $O_{j}$ et donc l'expression $\mathrm{i}: \mathrm{C} \stackrel{\sqsubseteq}{\leftrightarrows}: \mathrm{D}$ n'est en aucun cas équivalente à $\mathrm{j}: \mathrm{D} \stackrel{\sqsupseteq}{\longrightarrow} \mathrm{i}: \mathrm{C}$, la première signifiant que 
i:C est plus spécifique que $j:$ D du point de vue de $O_{j}$, la seconde signifiant que $\mathrm{j}: \mathrm{D}$ est plus générale que $i: C$ du point de vue de $O_{i}$ ou encore que $i: C$ est plus spécifique que $\mathrm{j}$ : D du point de vue de $O_{i}$.

L'interprétation distribuée $\mathfrak{I}$ d'un espace de contextes est donnée par un ensemble de relations de domaine de la forme $r_{i j} \subseteq \Delta^{\mathcal{I}_{i}} \times \Delta^{\mathcal{I}_{j}}$. Une telle relation détermine, pour tout objet $x$ de $\Delta^{\mathcal{I}_{i}}$, l'objet $y$ de $\Delta^{\mathcal{I}_{j}}$ auquel $x$ correspond. La notation $r_{i j}\left(\mathrm{C}^{\mathcal{I}_{i}}\right)$ dénote l'interprétation de la classe i:C de $O_{i}$ telle qu'elle est considérée dans le domaine d'interprétation de $O_{j}$. Ainsi, la sémantique d'une passerelle est donnée par une relation de domaine : I satisfait $\mathrm{i}: \mathrm{C} \stackrel{\sqsubseteq}{\longrightarrow} \mathrm{j}: \mathrm{D}$ si et seulement $\operatorname{si} r_{i j}\left(\mathrm{C}^{\mathcal{I}_{i}}\right) \subseteq \mathrm{D}^{\mathcal{I}_{j}}$ et I satisfait $\mathrm{i}: \mathrm{C} \stackrel{\sqsupseteq}{\longrightarrow} \mathrm{j}: \mathrm{D}$ si seulement $\operatorname{si} r_{i j}\left(\mathrm{C}^{\mathcal{I}_{i}}\right) \supseteq \mathrm{D}^{\mathcal{I}_{j}}$.

Un autre type de passerelle est utilisé pour spécifier une mise en correspondance entre individus : $\mathrm{i}: \mathrm{a} \stackrel{\equiv}{\longrightarrow} \mathrm{j}: \mathrm{b}$ signifie que l'individu $\mathrm{i}: \mathrm{a}$ de $O_{i}$ correspond à l'individu $\mathrm{j}: \mathrm{b}$ de $O_{j}$. Formellement, $\mathfrak{I}$ satisfait $\mathrm{i}: \mathrm{a} \stackrel{\equiv}{\longrightarrow} \mathrm{j}: \mathrm{b}$ si et seulement $\operatorname{si} r_{i j}\left(\mathrm{a}^{\mathcal{I}_{i}}\right)=\mathrm{b}^{\mathcal{I}_{j}}$.

\subsection{Le raisonnement global avec $\mathrm{C}$-OWL}

En complément des inférences associées à OWL, mises en œuvre localement dans chaque contexte, C-OWL permet d'exploiter un mécanisme de raisonnement global qui dépend des passerelles (Serafini et al., 2005). Dans ce dernier article d'ailleurs, il est fait une distinction entre "mécanisme de raisonnement local», qui fait référence aux inférences classiques des logiques de descriptions réalisées localement dans un contexte et le «mécanisme de raisonnement global», qui permet de réaliser des inférences d'un contexte à l'autre en s'appuyant sur les passerelles entre les contextes. Ainsi, le test de subsomption globale repose sur le principe de propagation de la subsomption, qui s'énonce de la façon suivante.

Si dans l'appariement $M_{i j}$ entre $O_{i}$ et $O_{j}$ sont déclarés $\mathrm{i}: \mathrm{A} \stackrel{\sqsupseteq}{\longrightarrow} \mathrm{j}: \mathrm{C}$ et $\mathrm{i}: \mathrm{B} \stackrel{\sqsubseteq}{\longrightarrow} \mathrm{j}: \mathrm{D}$, la donnée de la relation $\mathrm{i}: \mathrm{A} \sqsubseteq \mathrm{B}$ implique que $\mathrm{j}: \mathrm{C} \sqsubseteq \mathrm{D}$.

Autrement dit, si l'interprétation distribuée $\mathfrak{I}$ satisfait $\mathrm{i}: \mathrm{A} \stackrel{\sqsupseteq}{\longrightarrow} \mathrm{j}: \mathrm{C}, \mathrm{i}: \mathrm{B} \stackrel{\sqsubseteq}{\longrightarrow} \mathrm{j}: \mathrm{D}$ et si $\mathfrak{I} \vDash \mathrm{i}: \mathrm{A} \sqsubseteq \mathrm{B}$ alors $\mathfrak{I}$ satisfait $\mathfrak{I} \vDash \mathrm{j}: \mathrm{C} \sqsubseteq \mathrm{D}:$ la subsomption dans un contexte $O_{j}$ peut être déduite de la subsomption dans un contexte $O_{i}$ et des passerelles de $O_{i}$ à $O_{j}$.

De façon identique, l'instanciation globale repose sur le principe de propagation de l'instanciation, qui est le parallèle pour les individus du principe de propagation de la subsomption (Serafini et al., 2005).

Si dans l'appariement $M_{i j}$ entre $O_{i}$ et $O_{j}$ sont déclarés $\mathrm{i}: \mathrm{C} \stackrel{\sqsubseteq}{\longrightarrow} \mathrm{j}: \mathrm{D}$ et $\mathrm{i}: \mathrm{a} \stackrel{\equiv}{\longrightarrow} \mathrm{j}: \mathrm{b}$ alors la donnée de la relation $i: C(a)$ implique que $j: D(b)$.

Autrement dit, si l'interprétation distribuée $\mathfrak{I}$ satisfait $i: C \stackrel{\sqsubseteq}{\longrightarrow} j: D, i: a \stackrel{\equiv}{\longrightarrow} j: b$, et $i: C(a)$, alors $\mathfrak{I}$ satisfait $\mathfrak{I} \vDash j: D(b)$ : il est possible de déduire qu'un individu est instance d'une classe dans un contexte $O_{j}$ en s'appuyant sur l'instanciation de l'individu dans un autre contexte $O_{i}$ et sur les passerelles entre $O_{i}$ et $O_{j}$. 


\subsection{Les applications de C-OWL}

(Bouquet et al., 2004) mentionnent une seule application pratique de C-OWL, précédemment présentée dans (Stuckenschmidt et al., 2004), qui porte sur l'alignement d'ontologies, ici la mise en correspondance par des appariements de trois ontologies du domaine médical, à savoir GALEN (Rector et al., 1993), TAMBIS (Baker et al., 1999) et le réseau sémantique de l'UMLS (Nelson et al., 2002). Avec cette application de C-OWL sur l'alignement d'ontologies, l'application présentée ci-après sur la représentation de points de vue en cancérologie dans le cadre du projet KASIMIR semble être, au moins à l'heure actuelle, la seconde application existante et opérationnelle de C-OWL.

\section{Des points de vue pour la cancérologie : la représentation de référentiels en C-OWL}

La cancérologie, comme beaucoup de domaines en médecine, met en jeu plusieurs disciplines. Les connaissances décisionnelles dans ce domaine, que ce soit les connaissances contenues dans les référentiels ou les connaissances d'adaptation, combinent plusieurs aspects, plusieurs façons de voir et ainsi plusieurs points de vue. Dans chaque point de vue relatif à une discipline se constitue une façon propre de représenter, d'organiser et d'utiliser les connaissances. Ainsi, un cancérologue s'appuiera sur des éléments de connaissances particuliers et sur des représentations différentes du patient selon qu'il doit établir un traitement chirurgical ou une chimiothérapie. Ces points de vue multiples sont d'ailleurs visibles dans la structure même des référentiels. Le référentiel pour le traitement du cancer du sein par exemple se divise en plusieurs modules ou parties, qui se focalisent sur une phase de traitement particulière - chimiothérapie préopératoire, chirurgie, radiothérapie et traitement complémentaire - impliquant une à deux disciplines de la cancérologie, parmi la chimiothérapie, la chirurgie, la radiothérapie et l'hormonothérapie. Un module définit son propre vocabulaire et doit fournir un type de recommandation particulier pour une phase donnée de la prise en charge du patient, en n'utilisant pour cela que les caractéristiques spécifiquement utiles à cette recommandation. Par ailleurs, les réunions de concertation pluridisciplinaires regroupent des experts de différentes disciplines. Ces spécialistes du cancer adaptent le contenu des référentiels pour traiter des cas où l'application directe du référentiel n'est pas satisfaisante. Un expert apporte sa propre part à la solution, selon la discipline qu'il représente, et il collabore avec les experts des autres disciplines dans la mise en place d'une solution globale et satisfaisante.

Dans ce qui suit, nous proposons une modélisation de cet état des choses de la réalité, qui repose sur une représentation à points de vue multiples des connaissances comprises dans les référentiels et utiles à la prise de décision en cancérologie. Cette représentation des connaissances est réalisée dans le cadre du formalisme C-OWL, ce qui nous permet, en outre, de donner les grandes lignes d'une méthodologie de représentation de points de vue multiples dans une logique de descriptions distribuée comme C-OWL pour une application à l'échelle du monde médical. 
Il reste à souligner que l'essentiel de ce travail de modélisation des connaissances et de conception d'une représentation à points de vue multiples est «manuel». Il n'a pas été fait usage d'une méthode de conception, que ce soit pour la modélisation des connaissances (Schreiber et al., 1999) ou pour la programmation (avec UML (Booch et al., 2005)). Ce travail aurait pu bénéficier cependant du support de démarche de conception de ce genre : par exemple, dans (Nassar, 2005), une extension du formalisme UML, nommé VUML, propre à la conception avec points de vue, offre une démarche et un outil pour concevoir un modèle de classes avec points de vue multiples dans le cadre de la modélisation d'un système logiciel. Il serait intéressant de faire le lien avec ce type de démarche et de voir comment elle pourrait être adaptée à ce qui nous préoccupe ici, à savoir la conception de points de vue multiples en représentation des connaissances et leur implantation avec la logique de descriptions distribuée C-OWL.

\subsection{Les principes de la représentation}

Habituellement, la construction d'une ontologie dans un domaine donné suppose la mise en place d'un consensus entre les différents acteurs du domaine, afin d'établir les éléments de connaissances qui sont partagés par l'ensemble des acteurs et qui doivent être représentés indépendamment de tout contexte d'utilisation (Guarino, 1997). Dans le cadre d'une représentation à points de vue multiples, l'idée est toute autre. Il s'agit de construire une représentation reposant sur des points de vue, où dans un point de vue sont représentées des connaissances utiles à une application ou à un groupe particulier de personnes, qui coexistent et collaborent avec d'autres personnes ou applications.

C-OWL permet de représenter les points de vue sous forme d'ontologies locales ou contextes. Les appariements entre contextes permettent l'échange et la réutilisation de connaissances d'un contexte à l'autre, combinant ainsi les points de vue en une représentation globale. La démarche pour modéliser et formaliser des points de vue multiples sur les connaissances avec C-OWL est ainsi différente de la démarche plus classique de la construction d'une ontologie «globale». Il ne faut pas chercher à établir un consensus, mais au contraire à distinguer les points de vue. Pour cela, trois étapes sont nécessaires (voir la vision globale donnée sur la figure 3) :

1) dans un premier temps déterminer les points de vue pertinents dans le domaine,

2) puis construire une ontologie locale pour un point de vue,

3) et enfin établir les appariements entre les ontologies locales, qui indiquent les correspondances entre les connaissances manipulées dans les différents points de vue.

Il est préférable qu'un tel processus de conception soit mené en collaboration avec des experts du domaine, où les experts sont choisis pour leur spécialité, l'ensemble des spécialités du domaine devant être représenté. Le concepteur de la base est lui chargé de superviser et d'enchaîner les différentes étapes pour mettre en œuvre la réalisation effective de la représentation des connaissances à points de vue multiples. 


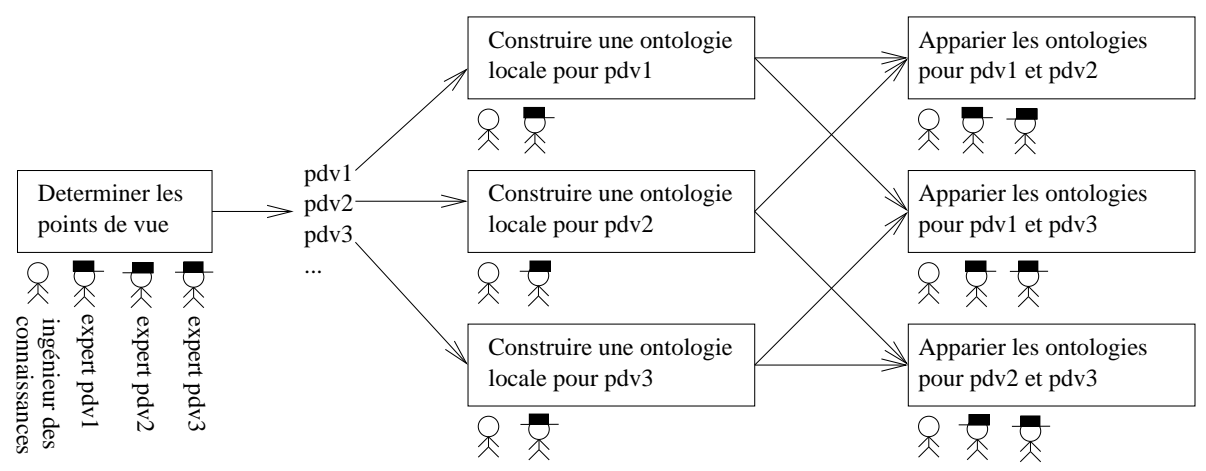

Figure 3. La démarche globale pour construire une représentation des connaissances à points de vue multiples

\section{Déterminer les points de vue pertinents}

Il s'agit lors de cette étape de déterminer quels sont les points de vue à représenter. Lorsqu'un domaine est suffisamment vaste et complexe, il est souvent organisé selon plusieurs services, plusieurs tâches, plusieurs groupes de travail ou encore plusieurs communautés (en accord avec le principe bien connu «diviser pour régner»). Cette organisation apporte une division a priori du domaine en points de vue, sur laquelle il est possible de s'appuyer. Par ailleurs, déterminer les points de vue est aussi l'occasion de distinguer les experts du domaine qui sont le mieux à même de modéliser les connaissances relatives à la spécialité qui donne naissance au point de vue. Dans cet ordre d'idée, la figure 3 donne une vision «idéale» : il est difficile de connaître tous les experts avant d'avoir déterminé les points de vue et, de façon duale, certains experts peuvent être impliqués dans la définition de plusieurs points de vue.

\section{Construire une ontologie locale}

Le principe ici est de construire une ontologie locale, en pratique un contexte formalisé en OWL, pour chaque point de vue qui vient d'être exhibé. Une telle ontologie locale doit comprendre les connaissances propres et utiles au point de vue considéré et cela indépendamment de tout autre point de vue. Il est important de chercher à ne couvrir que la partie du domaine qui est pertinente relativement au point de vue considéré, pour obtenir une représentation concise et homogène, au plus proche de la vision des spécialistes.

\section{Apparier les ontologies}

Dans cette dernière étape, les relations qu'entretiennent implicitement les points de vue entre eux sont rendues explicites grâce à des appariements entre les ontologies locales. Il faut souligner encore une fois que les appariements sont directionnels en C-OWL : une passerelle est un élément de connaissance ayant une origine et une ex- 
trémité, l'origine étant définie dans un contexte et l'extrémité dans un autre contexte, qui n'est utilisable que relativement à ces deux points de vue. La question à se poser pour un point de vue donné $O_{i}$ est celle de connaître quels sont les éléments de connaissances dans un ou plusieurs autres points de vue $O_{j}$ que $O_{i}$ est en mesure de « voir» et de réutiliser. Il faut ensuite établir les correspondances indiquant l'interprétation devant être faite des éléments de connaissances qui sont extérieurs à l'ontologie locale.

L'étape d'appariement dans la conception de points de vue multiples est pour le moment manuelle. Elle pourrait être dans certains cas semi-automatisée en utilisant des techniques de classification par treillis par exemple (Barbut et al., 1970; Ganter et al., 1999). De telles techniques permettraient sur la base des propriétés partagées par les classes de connaître les relations qui existent entre les extensions des classes (les ensembles d'instances) et donc d'établir à partir de là les passerelles associées.

\subsection{La représentation du référentiel du traitement du cancer du sein en $C$-OWL}

Dans cette section, nous illustrons les différentes phases explicitées ci-avant pour mettre en œuvre une représentation à points de vue multiples en C-OWL du référentiel relatif au traitement du cancer du sein. Ce référentiel fait partie en réalité d'un document plus volumineux couvrant le diagnostic du cancer du sein, accessible sur le site web du réseau Oncolor (Réseau de santé Oncolor (RSO), 2005e). L'exemple du cancer du sein a été choisi en raison de sa complexité et de son importance, en tout cas au niveau de la représentation de connaissances à points de vue multiples. Toutefois, il faut préciser que les exemples ci-après sont simplifiés, volontairement et obligatoirement, à cause de la complexité des connaissances du monde réel mises en jeu.

\subsubsection{Déterminer les points de vue pertinents}

Déterminer les points de vue pertinents est une tâche qui peut s'avérer difficile dans certains domaines. Il s'agit de trouver des divisions élémentaires dans les connaissances du domaine, indépendantes les unes des autres, mais collaborant entre elles. Ici, le référentiel est divisé en différentes parties, qui portent un intérêt sur différentes phases du traitement et qui impliquent différentes disciplines.

1) La chimiothérapie néo-adjuvante est un traitement de chimiothérapie recommandé avant la chirurgie et dont l'objectif principal est de freiner voire endiguer la progression de la tumeur, afin de favoriser un geste chirurgical moins important.

2) La chirurgie est en quelque sorte la phase centrale du traitement du cancer du sein. Il s'agit ici de décider quel traitement chirurgical va être employé en fonction des caractéristiques du patient et de sa maladie.

3) La radiothérapie complète la chirurgie en éliminant les éventuelles cellules envahies encore présentes, en particulier dans les ganglions. L'objectif est de décider quelles sont les parties de l'anatomie du patient qu'il est utile ou nécessaire d'irradier. 
4) Le traitement médical adjuvant fait partie des traitements complémentaires, qui peuvent s'intégrer à un traitement en chimiothérapie ou un traitement en hormonothérapie.

Ces parties du référentiel sont relativement indépendantes les unes des autres, bien qu'elles partagent certaines notions et fassent parfois référence à des décisions prises dans d'autres parties. Cette division du référentiel est par ailleurs utilisée quotidiennement par les médecins et apparaît plutôt bien adaptée à l'utilisation qui est la leur. Il semble donc pertinent de s'appuyer sur cette organisation a priori des connaissances pour déterminer les points de vue dans la représentation des connaissances du référentiel.

Le choix aurait pu être fait de considérer la partie traitement médical adjuvant comme deux points de vue différents, un pour la chimiothérapie et l'autre pour l'hormonothérapie, voire encore de regrouper les deux types de chimiothérapie, chimiothérapie néo-adjuvante et chimiothérapie complémentaire. Mais il est apparu préférable de conserver la structure initiale du référentiel. Dans la suite, c'est donc quatre points de vue qui vont être pris en compte : la chimiothérapie néo-adjuvante, la chirurgie, la radiothérapie et le traitement médical adjuvant. Ces quatre points de vue vont être représentés par quatre contextes en C-OWL, dénotés respectivement par $O_{c n a}, O_{c h i r}$, $O_{\text {radio }}$ et $O_{\text {tma }}$.

\subsubsection{Construire les ontologies locales}

\section{Les patients et les traitements associés}

Les quatre points de vue ou contextes considérés dans la suite ont en commun de recommander un traitement du cancer du sein (les termes «point de vue» et «contexte» sont utilisés indifféremment ci-après). Les classes Patient et Traitement qui représentent respectivement les patients et les traitements sont présentes dans chacun des quatre points de vue. Néanmoins, ces classes ne comprennent pas nécessairement les mêmes éléments dans chacun des points de vue, car, comme il se doit, elles ne sont pas interprétées de la même façon selon le point de vue. Par exemple, un patient dans $O_{\text {chir }}$ est une personne atteinte d'un cancer du sein, tandis qu'un patient dans $O_{t m a}$ est nécessairement une femme ${ }^{1}$ atteinte d'un cancer du sein et pour laquelle un traitement chirurgical a déjà été proposé. De la même façon, seuls les traitements de radiothérapie sont considérés dans $O_{\text {radio }}$ tandis qu'un traitement est nécessairement chirurgical dans $O_{c h i r}$. Le lien existant entre un patient et un traitement recommandé par le référentiel est matérialisé dans tous les points de vue par la propriété recommandation.

Dans une optique de résolution de problèmes, les instances de la classe Patient correspondent à des descriptions de problèmes particuliers et les instances de la classe Traitement correspondent aux solutions : un patient atteint d'un cancer du sein présente un problème et soigner ce patient nécessite de trouver une solution au problème.

1. Certains traitements d'hormonothérapie proposés dans le référentiel de traitement du cancer du sein ne sont applicables qu'aux femmes. 
Chaque point de vue est constitué de connaissances qui sont utilisées pour résoudre un certain type de problème - un problème local - répondant à une question particulière, comme par exemple «Quelle est la chirurgie recommandée ?», et cela, dans le cadre d'un problème global, qui est ici : «Quel est le traitement recommandé ?».

Le référentiel se présente sous la forme de diagrammes, similaires à des arbres de décision, associant aux caractéristiques d'un patient les recommandations à appliquer (voir figure 4). Ces diagrammes constituent une représentation «semi-formelle» de la marche à suivre pour proposer un traitement. Une branche d'un diagramme correspond à un ensemble de conditions caractérisant un patient. L'ensemble des patients caractérisés par un chemin particulier dans le diagramme - les conditions associées à la branche sont vérifiées par les patients - constitue une classe de patients partageant ces caractéristiques communes. Une telle classe peut alors être représentée par une classe en OWL, dont la définition s'appuie sur les caractéristiques et les contraintes associées qui déterminent le chemin considéré dans le diagramme. Par exemple, soit la classe $\mathrm{C}$ dont la définition en OWL est donnée par :

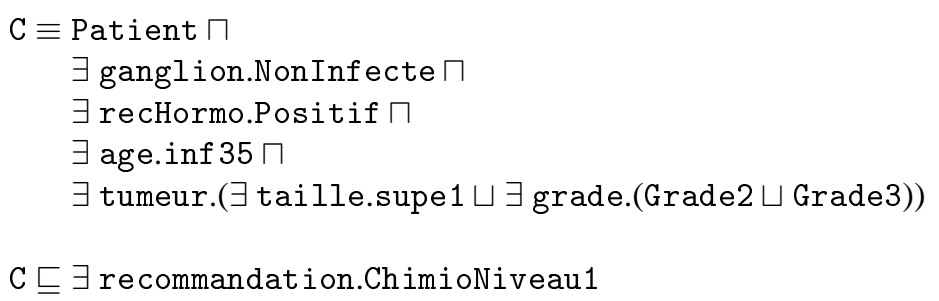

La classe C correspond au chemin d'origine «Statut ganglionnaire» et allant jusqu'au nœud «Chimiothérapie Niveau 1 » dans le diagramme de la figure 4. Le lien entre la classe de patients $\mathrm{C}$ et le traitement associé à ces patients est déclarés par l'intermédiaire de la propriété recommandation dans l'axiome où $\mathrm{C}$ figure en partie gauche : ainsi, en accord avec le référentiel, tout individu reconnu comme une instance de la classe $\mathrm{C}$ se voit recommandé une chimiothérapie de niveau 1.

Le point de vue $O_{\text {cna }}$

La chimiothérapie néo-adjuvante correspond à un ensemble de cures de chimiothérapie (suivant le protocole dit «FEC $100 »$ ), dont les résultats sont évalués au bout de la troisième cure (Réseau de santé Oncolor (RSO), 2005a). La décision à prendre porte sur la poursuite des cures ou non, en fonction de l'évolution de la tumeur. Si la tumeur régresse ou se stabilise, la chimiothérapie doit être poursuivie. Sinon, le passage du patient en réunion de concertation pluridisciplinaire (RCP) doit être envisagé. Il s'agit aussi d'évaluer si la réponse à la chimiothérapie est suffisante pour permettre une chirurgie conservatrice, c'est-à-dire une ablation partielle qui épargne les parties essentielles du sein. Par exemple, considérons l'extrait suivant du contexte $O_{c n a}$ :

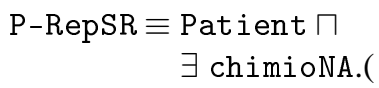




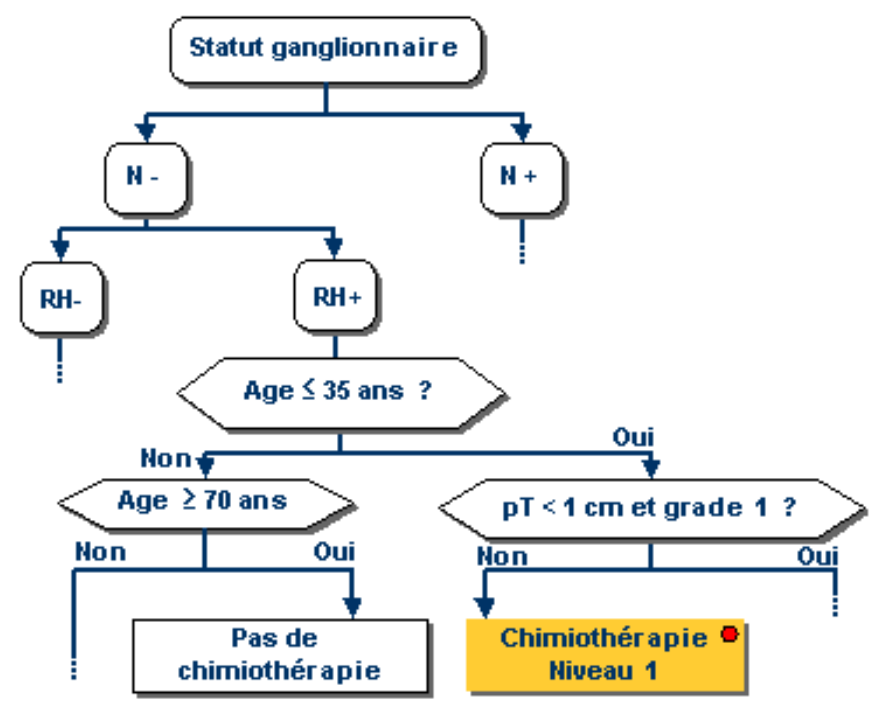

Figure 4. Un extrait du référentiel de traitement du cancer du sein

(ChimioNeoAdjuvante $\square$

$\exists$ reponse.(Regression $\sqcup$ Stabilisation)

)

P-RepSR $\sqsubseteq \exists$ recommandation.PoursuiteChimio

La classe P-RepSR représente les patients ayant subi un traitement par chimiothérapie néo-adjuvante et pour lesquels la réponse au traitement est une stabilisation ou une régression du cancer. Le référentiel recommande dans ce cas de poursuivre la chimiothérapie, ce qui est indiqué par le dernier axiome introduisant la classe PoursuiteChimio. Les classes Stabilisation ou Régression possèdent de plus une propriété à valeur booléenne dénotée suffisante qui indique si oui ou non la tumeur a suffisamment évolué pour autoriser une chirurgie conservatrice.

Le point de vue $O_{\text {chir }}$

La chirurgie est le traitement central dans la prise en charge d'un patient atteint d'un cancer du sein. Pour simplifier, seules les connaissances décisionnelles associées à la chirurgie des tumeurs opérables d'emblée (Réseau de santé Oncolor (RSO), 2005b) sont décrites ici, qui sont parmi les situations les moins complexes à traiter. Il s'agit dans ce cas de choisir entre une mastectomie dite partielle et une mas- 
tectomie dite de Patey. Une mastectomie partielle correspond à une ablation conservatrice du sein, se concentrant uniquement sur la tumeur. Elle est nécessairement associée à un curage axillaire des deux étages inférieurs, ce qui correspond au prélèvement de ganglions dans le but de les analyser. L'indication des deux étages inférieurs est en rapport avec l'endroit où les ganglions doivent être prélevés. Une mastectomie de $\mathrm{Pa}$ tey correspond à une ablation totale du sein, qui est associée à un curage axillaire complet, c'est-à-dire des trois étages. Par exemple, un patient ayant une tumeur opérable d'emblée et non multifocale est traité par une mastectomie partielle, ce qui se représente par les axiomes suivants dans $O_{\text {chir }}$ :

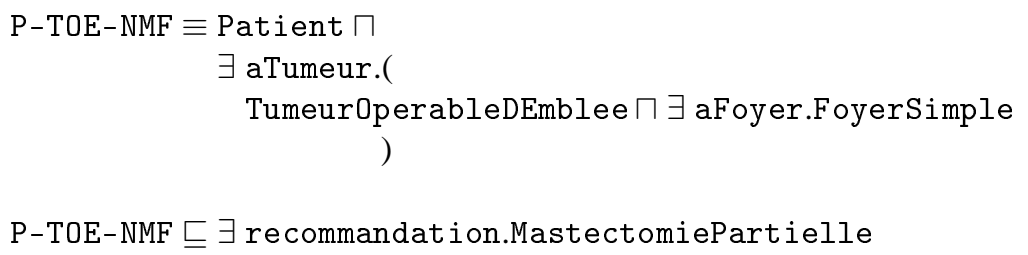

Le point de vue $O_{\text {radio }}$

La radiothérapie a pour objectif de compléter la chirurgie en éliminant les cellules envahies éventuellement résiduelles, en particulier dans les ganglions. Il s'agit alors de déterminer la ou les zones qu'il est utile d'irradier dans le sein : la paroi de la zone prélevée par une mastectomie partielle, la zone sus-claviculaire ou la chaîne mammaire interne du sein (CMI), éventuellement élargie (CMIE) (Réseau de santé Oncolor (RSO), 2005c).

Les caractéristiques du patient prises en compte lors de cette décision concernent la chirurgie proposée, la localisation de la tumeur dans le sein, sa taille, la présence d'une atteinte cutanée et de cellules envahies dans les ganglions. Ainsi, les axiomes suivants indiquent qu' un patient ayant subi une chirurgie conservatrice, dont les ganglions sont non envahis et dont la tumeur est localisée dans le quadrant inféro-interne du sein, doit être traité par une radiothérapie du sein et de la chaîne mammaire interne élargie.

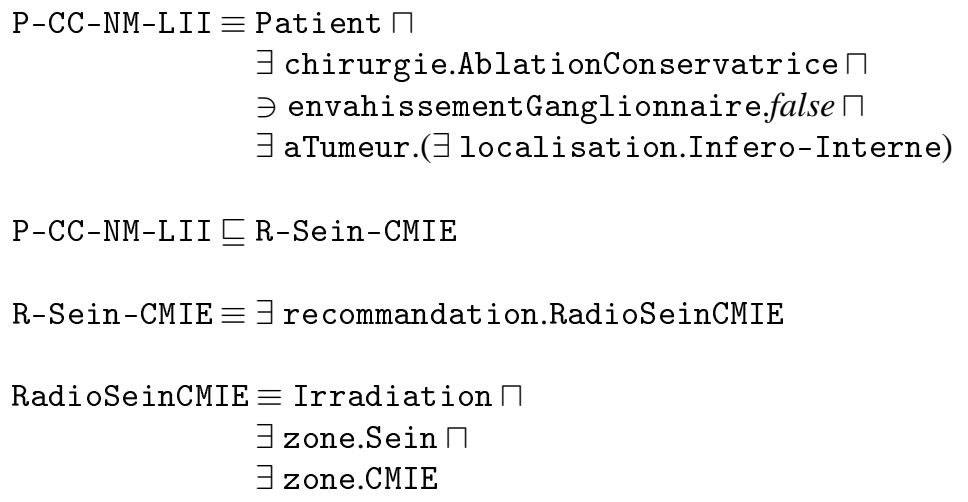


Il faut remarquer qu'il est possible de recommander de ne pas faire de radiothérapie à l'aide d'un axiome comme le suivant, qui indique que les patients représentés par des instances de la classe $P$ ne peuvent se voir recommander une radiothérapie.

$$
\mathrm{P} \sqsubseteq \neg \exists \text { recommandation.Radiothrapie }
$$

Le point de vue $O_{\text {tma }}$

La partie la plus importante (en taille) du référentiel de traitement du cancer du sein concerne le traitement médical adjuvant (Réseau de santé Oncolor (RSO), 2005d). Elle porte sur la recommandation d'une hormonothérapie ou d'une chimiothérapie qui vient compléter la chirurgie. L'hormonothérapie repose sur le contrôle des hormones ayant une influence sur le développement de la tumeur. Il y a deux hormonothérapies utilisées dans le cadre du traitement du cancer du sein :

- l'hormonothérapie par suppression consiste à supprimer les fonctions des ovaires, productrices d'hormones, de façon chimique ou chirurgicale (ablation des ovaires) ;

- l'hormonothérapie par addition consiste à administrer une cure de Tamoxifène, qui est un produit anti-œstrogènes. Les chimiothérapies envisageables comprennent 4 à 6 cures d'injections selon les protocoles FEC 50 (chimiothérapie de niveau 1) ou FEC 100 (chimiothérapie de niveau 2).

Le choix du traitement médical adjuvant est guidé par des caractéristiques telles que l'état des récepteurs hormonaux, le nombre de ganglions envahis, l'âge du patient, la taille de la tumeur et son grade (1,2 ou 3). Le référentiel indique par exemple qu'un patient âgé de moins de 35 ans, n'ayant pas de ganglion envahi, des récepteurs hormonaux positifs, une tumeur de grade supérieur ou égal à 2 ou de taille supérieure ou égale à $1 \mathrm{~cm}$, doit être traité par une chimiothérapie de niveau 1 :

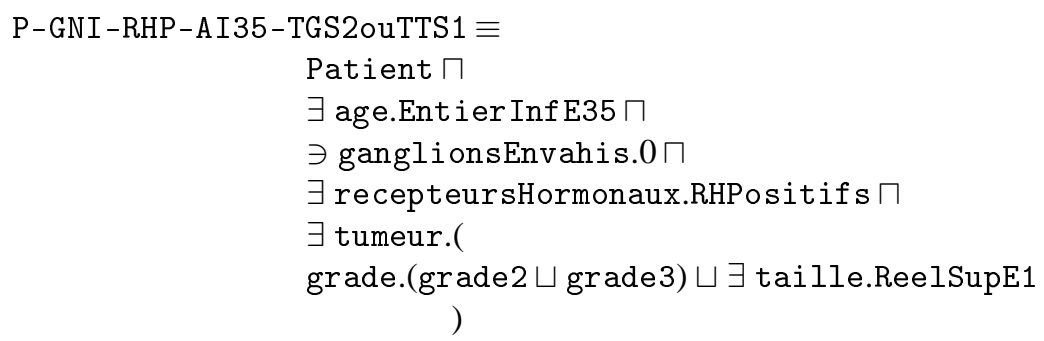

Les classes EntierInfE35 et RéelSupE1 correspondent à des types de données représentant respectivement les entiers inférieurs ou égaux à 35 et les réels supérieurs ou égaux à 1. En outre, le statut des récepteurs hormonaux est défini en fonction du statut des récepteurs aux œustrogènes et à la progestérone par les les axiomes ci-après : 


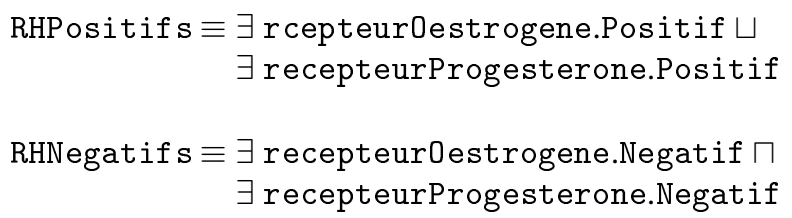

\subsubsection{Apparier les différents points de vue}

Dans cette dernière étape, les relations pouvant exister entre les quatre points de vue, $O_{\text {cna }}, O_{\text {chir }}, O_{\text {radio }}$ et $O_{\text {tma }}$, sont exprimées sous forme d'appariements. Chaque ontologie locale représentée correspond à la vision d'une discipline de la cancérologie impliquée dans une phase particulière du traitement. Les différentes phases du traitement s'inscrivent à différents moments dans la prise en charge du patient. Ainsi, la chirurgie ayant lieu après la chimiothérapie néo-adjuvante, il est naturel que les décisions en chirurgie suivent et s'appuient sur les décisions prises pour la chimiothérapie néo-adjuvante. De la même façon, les points de vue de la radiothérapie et du traitement médical adjuvant peuvent échanger des informations avec le point de vue de la chirurgie. Ces relations ne sont pas toujours faciles à mettre en œuvre. En effet, puisque la chimiothérapie néo-adjuvante intervient nécessairement avant la chirurgie, il n'y a aucune raison pour que les connaissances décrites dans $O_{\text {chir }}$ aient une influence sur celles de $O_{c n a}$. De la même façon, les points de vue $O_{\text {radio }}$ et $O_{\text {tma }}$ renferment des connaissances qui sont utiles après la décision de traitement chirurgical : les éléments de connaissances dans $O_{\text {chir }}$ doivent donc être indépendants de ceux décris dans $O_{\text {radio }}$ et $O_{t m a}$.

La figure 5 résume les appariements qu'il est pertinent de mettre en œuvre en fonction des connaissances du domaine et de leurs relations. Sur cette figure, une flèche allant d'un contexte $O_{i}$ vers un contexte $O_{j}$ indique la mise en place d'un appariement $M_{i j}$. Ces appariements vont être réifiés par les deux types de passerelles, en-dessous $(\stackrel{\sqsubseteq}{\longrightarrow})$ et au-dessus $(\stackrel{\sqsupseteq}{\longrightarrow})$, reliant les classes des contextes représentés.

L'appariement de $O_{\text {cna }}$ vers $O_{\text {chir }}$

Tout patient en chimiothérapie néo-adjuvante sera nécessairement, par la suite un patient en chirurgie. En revanche, un patient en chirurgie n'a pas nécessairement été un patient en chimiothérapie néo-adjuvante. Ainsi, l'appariement de $O_{c n a}$ vers $O_{c h i r}$ contient la passerelle :

cna:Patient $\stackrel{\sqsubseteq}{\longrightarrow}$ chir:Patient

Par ailleurs, la chimiothérapie néo-adjuvante a pour objectif de réduire la taille de la tumeur afin de permettre une mastectomie partielle, donc de rendre la tumeur opérable d'emblée. Par suite, des passerelles doivent permettre d'inférer que la tumeur peut être considérée comme opérable d'emblée dans $O_{c h i r}$, en fonction du résultat de la chimiothérapie néo-adjuvante indiqué dans le point de vue $O_{c n a}$ : 


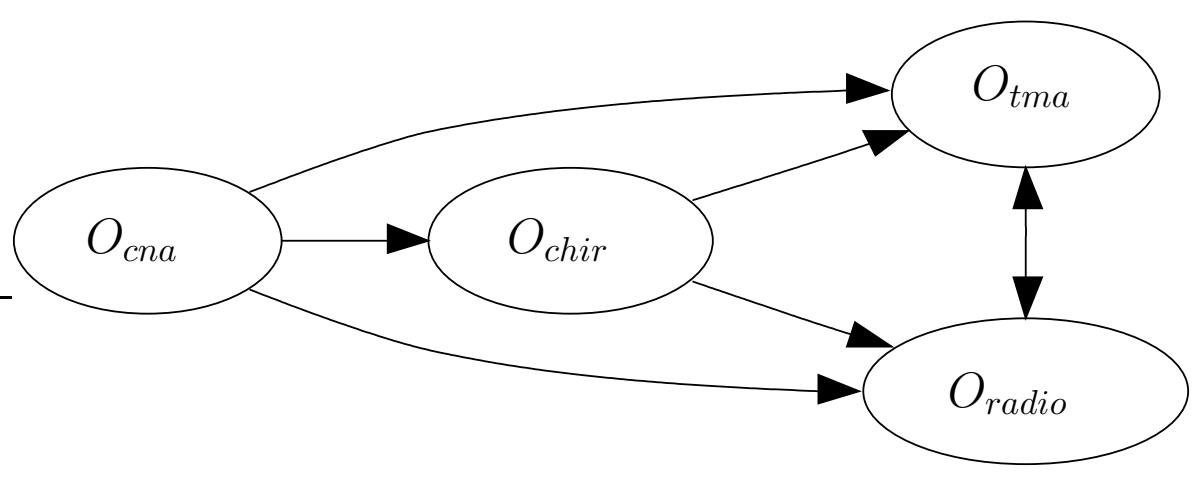

Figure 5. Les appariements mis en cuvre dans la représentation du référentiel de traitement du cancer du sein

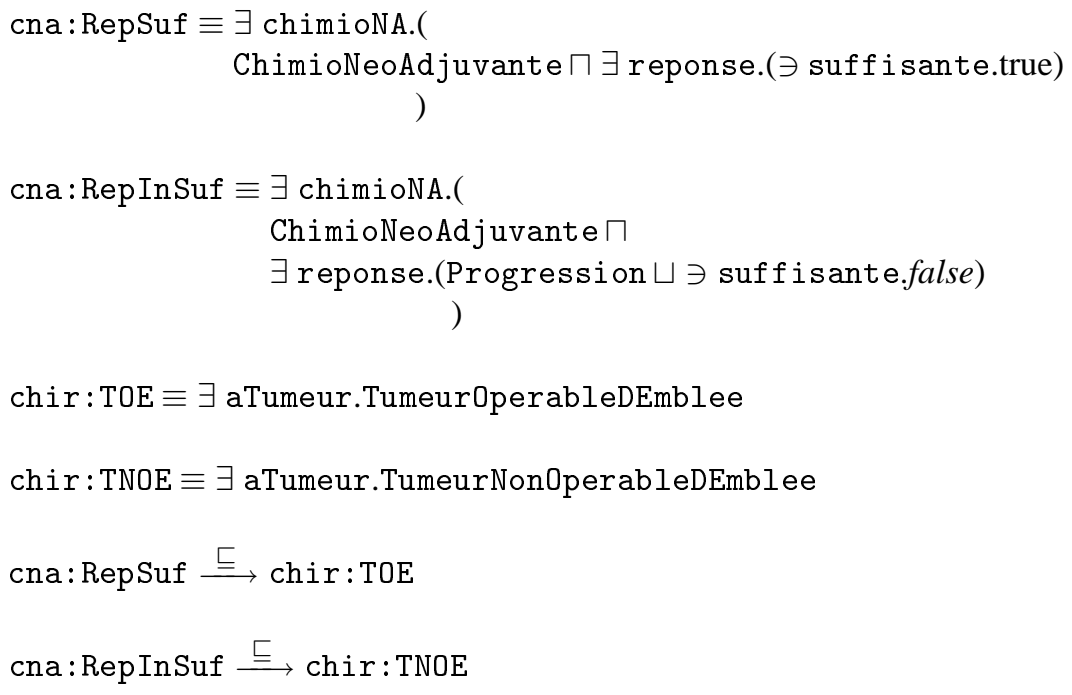

L'appariement de $O_{\text {chir }}$ vers $O_{\text {radio }}$

La question du traitement de radiothérapie ne se pose que pour les patients ayant subi une chirurgie. Ainsi, l'appariement du point de vue $O_{\text {chir }}$ vers le point de vue $O_{\text {radio }}$ s'appuie sur la passerelle :

$$
\text { chir:Patient } \stackrel{\sqsupseteq}{\longrightarrow} \text { radio:Patient }
$$

Les décisions en radiothérapie reposent sur les décisions prises lors du traitement chirurgical et une des caractéristiques du patient prises en compte est la chirurgie ef- 
fectuée. Un patient pour qui une recommandation de mastectomie partielle est proposée en chirurgie est considéré, dans le contexte de la radiothérapie, comme un patient ayant subi une chirurgie conservatrice. Ainsi, les passerelles suivantes sont comprises dans l'appariement de $O_{\text {chir }}$ vers $O_{\text {radio }}$ :

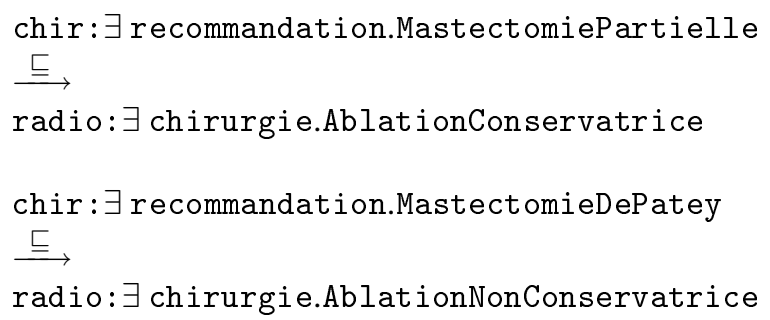

L'appariement de $O_{\text {chir }}$ vers $O_{\text {tma }}$

De la même façon que pour la radiothérapie, un patient pour lequel la question du traitement médical adjuvant se pose est un patient pour lequel une chirurgie a été proposée. Ainsi, l'appariement de $O_{c h i r}$ vers $O_{t m a}$ contient la passerelle :

chir:Patient $\stackrel{\sqsupseteq}{\longmapsto}$ tma:Patient

L'appariement de $O_{\text {cna }}$ vers $O_{\text {radio }}$ et vers $O_{t m a}$

Il est établi dans le référentiel que tout patient ayant subi une chimiothérapie néoadjuvante doit être traité en radiothérapie comme si le statut ganglionnaire était positif. Pour simplifier, cette règle est représentée dans l'appariement entre $O_{c n a}$ et $O_{\text {radio }}$ par la passerelle suivante :

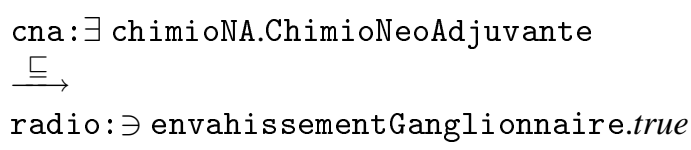

La chimiothérapie est un traitement qui ne peut être effectué à trop grandes doses pour un patient. Ainsi, le référentiel indique qu'un patient ayant été traité par chimiothérapie néo-adjuvante ne doit pas être traité ensuite par chimiothérapie dans le cadre du traitement médical adjuvant. Ce qui est représenté par la passerelle :

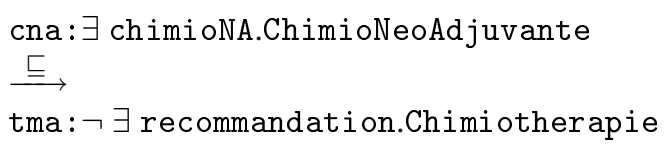


Les appariements entre $O_{\text {tma }}$ et $O_{\text {radio }}$

Les décisions prises en radiothérapie n'ont pas d'influence sur celles concernant le traitement médical adjuvant et vice versa. Néanmoins, certaines caractéristiques des patients, comme le statut ganglionnaire ou la taille de la tumeur, sont représentées dans les deux contextes $O_{\text {radio }}$ et $O_{t m a}$. Les passerelles servent ici à partager des informations sur les patients d'un contexte à l'autre. Les passerelles associées à l'appariement de $O_{t m a}$ vers $O_{\text {radio }}$ permettent de compléter la description du patient sur le statut ganglionnaire en radiothérapie en fonction de l'information sur le nombre de ganglions envahis telle qu'elle est représentée dans le point de vue du traitement médical adjuvant.

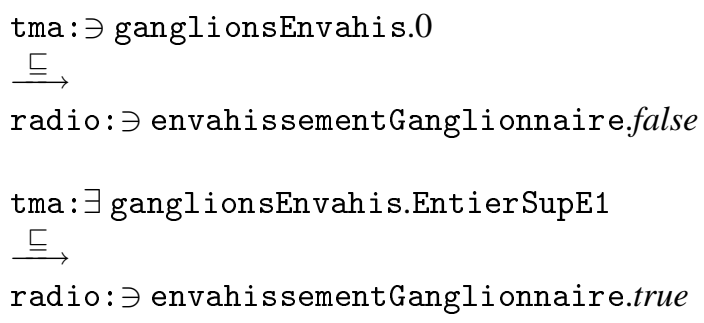

Ci-dessus, EntierSupE1 dénote le type de données représentant les entiers supérieurs ou égaux à 1. Les passerelles inverses font l'objet de l'appariement de $O_{t m a}$ vers $O_{\text {radio }}$ et permettent le même genre d'échange de connaissances dans l'autre sens.

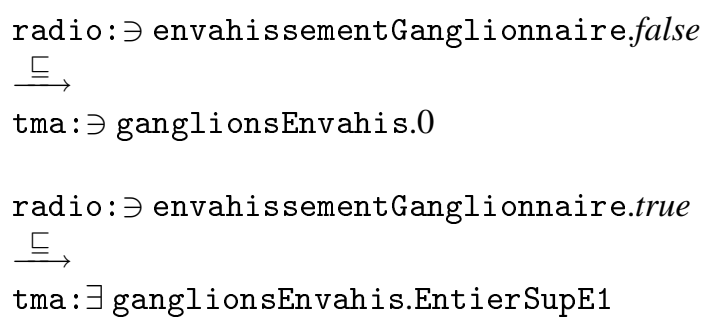

\subsection{Le raisonnement en fonction de points de vue multiples}

L'instanciation globale est le principal mécanisme de raisonnement pour l'aide à la décision, qui tire parti des points de vue multiples. Un exemple concret sur le traitement du cancer du sein va illustrer la façon dont ce mécanisme permet de conduire à la solution d'un problème en combinant des points de vue multiples. Soit le problème constitué par le cas d'une patiente âgée de 33 ans et atteinte d'un cancer du sein. Les caractéristiques de la tumeur de cette patiente sont les suivantes : la tumeur n'est pas multifocale, elle est opérable d'emblée, de grade 2, elle est localisée dans le quadrant 
inféro-interne du sein et mesure $1,8 \mathrm{~cm}$, les récepteurs hormonaux sont positifs et le curage ne révèle pas de ganglion envahi.

Le médecin cherchant à établir un traitement peut considérer les caractéristiques de cette patiente selon un ou plusieurs des quatre points de vue définis précédemment, à savoir $O_{c n a}, O_{c h i r}, O_{\text {radio }}$ et $O_{t m a}$. Dans cet exemple, la chimiothérapie néo-adjuvante n'intervient pas et c'est donc les recommandations des référentiels en rapport avec la chirurgie, la radiothérapie et le traitement médical adjuvant qui sont prises en compte. La patiente est donc représentée par trois instances locales de la classe des patients, dénotées par chir:p, radio:p et tma:p. Différentes assertions sont ensuite déclarées dans les différents points de vue pour traduire les caractéristiques de la patiente introduites plus haut.

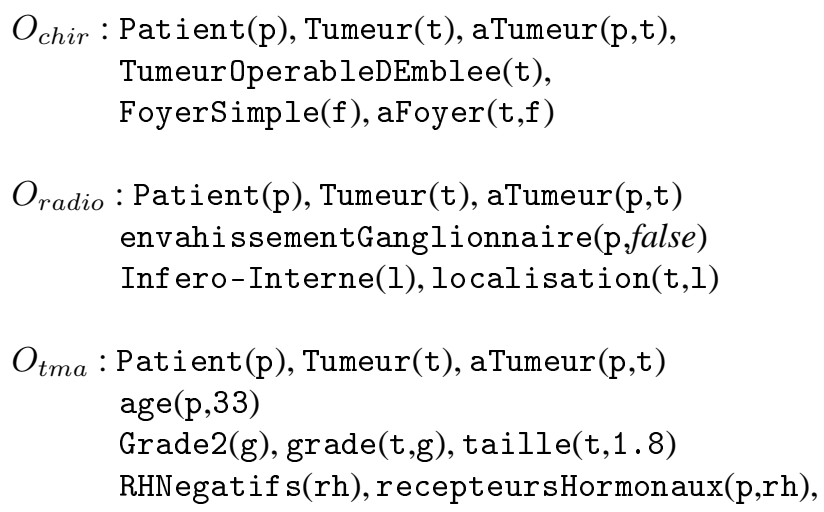

Des passerelles d'équivalence sont déclarées entre les instances des différents points de vue pour indiquer que ces instances représentent la même patiente :

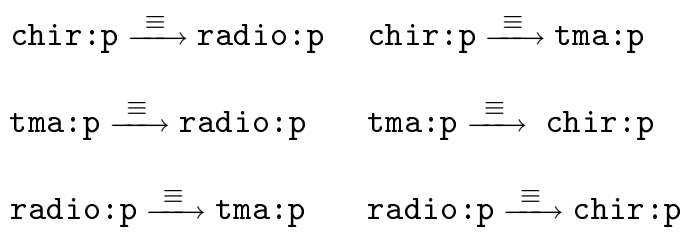

Sur la base des assertions introduites dans $O_{\text {chir }}$ et des axiomes associés à la représentation du point de vue $O_{\text {chir }}$, les inférences suivantes sont réalisées.

1) Point de vue $O_{\text {chir }}$

- l'instance $\mathrm{p}$ est reconnue comme une instance de la classe P-TOE-NMF : la tumeur est opérable d'emblée et le foyer est simple;

- à la suite, une mastectomie partielle est recommandée.

2) Point de vue $O_{\text {radio }}$

- la première passerelle de $O_{\text {chir }}$ vers $O_{\text {radio }}$ permet de compléter les connaissances sur $\mathrm{p}$ : l'ablation peut être conservatrice; 
- la localisation de la tumeur et le statut de l'envahissement ganglionnaire permettent d'inférer que $\mathrm{p}$ est une instance de la classe P-CC-NM-LII, subsumée par la classe R-Sein-CMIE, pour laquelle une radiothérapie du sein et de la chaîne mammaire interne élargie est alors recommandée (RadioSeinCMIE).

3) Point de vue $O_{t m a}$

- la première passerelle de $O_{\text {radio }}$ vers $O_{t m a}$ permet de compléter encore les connaissances sur $\mathrm{p}$ : il n'existe pas de ganglions envahis ;

- l'âge de la patiente, l'état des récepteurs hormonaux (RH positifs), le grade de la tumeur et sa taille permettent d'inférer que $\mathrm{p}$ est une instance de la classe P-GNI-RHP-AI35-TGS2ouTTS1, pour laquelle une chimiothérapie de niveau 1 est recommandée.

Finalement, la solution recommandée par le référentiel est représentée par trois instances de classes de traitements, c'est-à-dire trois points de vue sur la solution, qui se complètent pour former une solution globale :

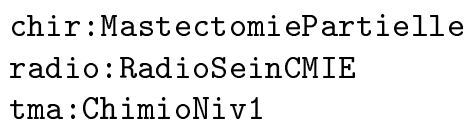

\subsection{Les apports d'une représentation à points de vue multiples}

Plusieurs critères peuvent être analysés pour mesurer les apports d'une représentation à points de vue multiples dans le traitement d'un problème complexe du monde réel, à l'image du problème qu'est l'aide à la décision dans le traitement du cancer du sein. Parmi ces critères ont été retenus les suivants, sachant qu'il n'est pas possible d'être exhaustif mais que ces critères semblent particulièrement représentatifs et importants :

- le pouvoir d'expression ou la richesse du langage de représentation;

- la facilité de conception en termes d'ingénierie des connaissances;

- la facilité de maintenance en termes d'évolution du système de représentation, pour l'adjonction, la suppression ou la mise à jour des connaissances de la base ;

- les possibilités d'inférence associées à la base de connaissances.

\section{Le pouvoir d'expression}

L'évaluation de ce premier critère cherchant à mesurer l'apport d'une représentation à points de vue multiples codée en C-OWL est sujette à discussion. D'un point de vue purement formel, le langage C-OWL ne permet pas d'exprimer plus de choses que OWL. En outre, il est montré dans (Borgida et al., 2002) qu'une base de connaissances codée dans une logique de descriptions distribuée peut être transformée sans autre perte d'information en une base de connaissances codée en logique de descriptions non distribuée, comme $\mathcal{S H I} \mathcal{L}$ par exemple (Baader et al., 2004). Ainsi, les ontologies 
locales d'un espace de points de vue en C-OWL peuvent être regroupée en une ontologie unique exprimée en OWL DL. Néanmoins, il existe en C-OWL le principe dit de non-propagation de l'incohérence (Bouquet et al., 2004 ; Serafini et al., 2005), qui n'est pas pris en compte dans la transformation développée dans (Borgida et al., 2002) (du distribué vers le non distribué). Ainsi, détecter une incohérence dans un point de vue en C-OWL ne doit pas avoir d'influence sur la cohérence dans les autres points de vue, que ce soit au niveau des expressions traitées comme au niveau des éléments représentés. A l'heure actuelle, ce principe n'a pas été directement utilisé dans l'application qui a été présentée : les points de vue sont a priori cohérents, localement autant que globalement, mais la possibilité reste ouverte : il peut s'avérer important de détecter l'incohérence d'une expression dans un point de vue qui ne peut pas l'être dans un autre et ainsi agir en conséquence : la quadrature du cercle ne pose aucun problème du point de vue de la couleur des éléments géométriques.

\section{La facilité de conception et de maintenance}

La représentation de points de vue avec C-OWL présente en revanche un intérêt indéniable en termes d'ingénierie et de gestion des connaissances. La démarche de construction d'une représentation à points de vue multiples consiste à construire un ensemble de représentations locales, chacune correspondant à une «vue » sur une partie du domaine considéré. Cette vue est réservée à une tâche, une application ou un groupe de personnes particulier. Les représentations locales sont ensuite appariées deux à deux. De cette façon, il n'est pas indispensable de chercher et mettre en œuvre un consensus global sur l'ensemble du domaine. Un point de vue se concentre sur un aspect précis, une représentation locale concise et homogène, qui peut prétendre de surcroît à l'exhaustivité (si nécessaire et autant que faire se peut) selon l'utilité et l'intérêt recherchés.

En parallèle, l'accès aux connaissances et la maintenance d'un ensemble de points de vue apparaît plus simple que dans une ontologie globale unique. Les acteurs d'un domaine, selon le point de vue qu'ils adoptent, ont la possibilité de se ramener uniquement aux contextes qu'ils jugent pertinents pour obtenir une représentation des connaissances du domaine adaptée à leur échelle et à leur usage. La collaboration et le partage de connaissances avec les autres points de vue sont réalisés par le biais des passerelles si nécessaire. Dans le même ordre d'idées, une évolution dans les connaissances dans un point de vue particulier n'est prise en compte que localement, sans interférer avec les autres points de vue.

Dans le cadre de l'exemple ci-avant portant sur le cancer du sein, une comparaison peut être faite avec un référentiel construit dans un premier temps sur la base d'une représentation unique, codée dans un langage de représentation par objets n'intégrant pas la notion de point de vue (d'Aquin et al., 2004a). La construction de cette dernière représentation a nécessité de faire cohabiter l'ensemble des expertises du domaine. En particulier, il s'est avéré nécessaire de devoir choisir entre différentes représentations possibles pour certains éléments : un tel choix, forcément arbitraire, ne correspond pas toujours voire jamais aux habitudes des acteurs du domaine. Par exemple, pour des besoins liés au traitement médical adjuvant, il a été décidé de représenter le statut 
ganglionnaire par le nombre de ganglions envahis, façon de faire en total désaccord avec la représentation habituelle faite en radiothérapie.

En outre, l'apparition d'une nouvelle technique, par exemple en chirurgie, suppose d'ajouter et de mettre à jour des éléments de connaissances parmi les 1000 classes et plus qui composaient la représentation du référentiel, une tâche qu'il est très difficile d'appréhender manuellement. Dans le cadre d'une représentation unique, une telle évolution peut potentiellement remettre en cause une grande partie de la représentation, y compris des éléments qui sont sans rapport avec la chirurgie. Dans une représentation à points de vue multiples du même référentiel, un point de vue apporte une représentation concise qui reste à l'échelle du spécialiste, composée de quelques dizaines de classes et qui évolue indépendamment des autres points de vue.

\section{Les possibilités d'inférence}

Les mécanismes de raisonnements comme la subsomption ou l'instanciation sont complexes en logique de descriptions (Baader et al., 2003) et donc en OWL. De fait, une des problématiques parmi les plus importantes à l'heure actuelle pour le web sémantique concerne le passage à l'échelle. Le formalisme C-OWL permet non seulement de construire des ontologies modulaires, mais aussi de mettre en œuvre des mécanismes de raisonnements locaux et distribués, où un moteur d'inférence est associé à chacun des points de vue de l'espace de contextes (Serafini et al., 2005). Les inférences globales combinent les connaissances de plusieurs contextes et sont réalisées de façon décentralisée, par le biais d'une communication «pair-à-pair», ici entre les différents moteurs d'inférence associés à chaque points de vue (Serafini et al., 2004). La complexité du raisonnement et les ressources nécessaires sont de cette manière distribuées entre les «moteurs d'inférence locaux», pouvant s'exécuter éventuellement sur des machines différentes et distantes. De plus, les points de vue d'un espace de contextes étant (relativement) indépendants les uns des autres, un moteur d'inférence local peut être optimisé pour une certaine tâche et pour ne prendre en compte que le niveau d'expression des connaissances nécessaire à la représentation dans le point de vue correspondant.

\section{Conclusion et perspectives}

Dans cet article, nous avons présenté une application de la représentation de connaissances avec points de vue multiples pour le traitement du cancer du sein. Cette représentation regroupe quatre points de vue provenant de différentes disciplines de la cancérologie, intervenant à différentes phases du traitement. Ainsi mis en place et implanté, l'ensemble de ces quatre points de vue constitue lui-même un point de vue plus global, qui peut coexister avec d'autres points de vue, par exemple les points de vue relatifs au diagnostic, à la surveillance ou aux essais cliniques dans le traitement du cancer du sein. Chaque représentation à points de vue multiples devient alors un «point de vue spécialisé » d'un point de vue englobant sur le traitement du cancer du sein en général. Par extension, un point de vue encore plus général pourrait être mis 
en œuvre qui serait celui de la prise en charge de patients atteints de cancer du sein en Lorraine. Un tel point de vue peut lui-même être mis en relation (apparié) avec un point de vue sur la prise en charge d'autres types de cancers ou avec la représentation de référentiels dans d'autres régions en France ou à l'étranger, etc.

Parmi les formalismes de représentation mentionnés en début d'article, deux permettent d'organiser les points de vue en hiérarchie : ceux décrits dans (Attardi et al., 1995) et dans (McCarthy, 1993). Dans (McCarthy, 1993), l'idée est développée que tout contexte $c$ est nécessairement placé dans un contexte englobant, qui explicite les éléments laissés implicites dans $c$ (les suppositions). Par exemple, en cancérologie, dans le point de vue du diagnostic, un patient est implicitement considéré comme quelqu'un pour qui un cancer du sein est suspecté. Dans le point de vue du traitement, un patient est une personne pour laquelle un cancer du sein a été diagnostiqué. A l'heure actuelle, les contextes d'un espace de contextes en C-OWL ne sont pas directement comparables entre eux (par exemple pour les organiser en une hiérarchie). Ainsi, un contexte englobant serait dans ce formalisme représenté au même niveau que ceux qu'il englobe. Il pourrait être intéressant de disposer d'un processus autorisant les comparaisons entre points de vue, et ainsi de pouvoir détecter les points de vue en relation de spécialisation, et de profiter de cette possibilité au niveau de la conception de l'ensemble des points de vue.

Le processus évoqué ci-avant reste prospectif et pose d'une certaine façon au moins une question et appelle une remarque pragmatique. La question est relative à la complexité du processus de points de vue englobant qu'il faudrait mettre en œuvre : où commencer, où s'arrêter et ensuite, comment gérer la complexité de l'emboîtement des points de vue? Le problème reste entier pour le moment. La remarque pragmatique porte sur le processus de conception, qui relève de ce qui pourrait être appelé une démarche ascendante de la construction d'ontologies. De façon duale, la démarche la plus courante est généralement descendante : il s'agit de construire une représentation la plus générale et la plus partagée possible, couvrant la plus grande part du domaine, avec l'éventualité ensuite de spécialiser la représentation globale pour des applications particulières. Ce qui a été montré ici consiste à l'inverse à considérer en premier lieu les éléments de connaissances locaux, les points de vue élémentaires, pour ensuite les combiner par l'intermédiaire des appariements, afin de construire une représentation globale constituée d'un ensemble de point de vue. La représentation globale obtenue couvre la globalité du domaine et se voit composée de représentations locales, directement opérationnelles. Pour pousser l'analogie plus loin, la démarche ascendante se voit associer plus de possibilités de construction semi-automatique que la démarche descendante, comme par exemple la classification par treillis (Barbut $e t$ al., 1970; Ganter et al., 1999). 


\section{Bibliographie}

Antoniou G., van Harmelen F., A Semantic Web Primer, The MIT Press, 2004.

Attardi G., Simi M., "A Formalization of Viewpoints", Fundamenta Informaticae, vol. 23, $n^{\circ}$ 2/3/4, p. 149-173, 1995.

Baader F., Calvanese D., McGuinness D., Nardi D., Patel-Schneider P. (eds), The Description Logic Handbook, Cambridge University Press, cambridge, UK, 2003.

Baader F., Horrocks I., Sattler U., "Description Logics”, S. Staab, R. Studer (eds), Handbook on Ontologies, Springer, Berlin, p. 3-28, 2004.

Baker P., Goble C., Bechhofer S., Paton N., Steven R., Brass A., "An ontology for bioinformatics applications", Bioinformatics, vol. 15, n 6, p. 510-520, 1999.

Barbut M., Monjardet B., Ordre et classification - Algèbre et combinatoire (2 tomes), Hachette, Paris, 1970.

Benerecetti M., Bouquet P., Ghidini C., "On the Dimensions of Context Dependence : Partiality, Approximation, and Perspective", Proc. of the Third International and Interdisciplinary Conference on Modeling and Using Context, vol. 2116 of Lecture Notes In Computer Science, Springer, p. 59-72, 2001.

Bobrow D., Winograd T., "An overview of KRL, a knowledge representation language", Cognitive Science, vol. 1, $\mathrm{n}^{\circ}$ 1, p. 3-46, 1977.

Booch G., Rumbaugh J., Jacobson I., The Unified Modeling Language User Guide, Addison Wesley, 2005. (Second Edition).

Borgida A., Serafini L., "Distributed description logics : Directed domain correspondences in federated information sources", Proc. of the Intenational Conference on Cooperative Information Systems, 2002.

Bouquet P., Giunchiglia F., van Harmelen F., Serafini L., Stuckenschmidt H., "Contextualizing Ontologies", Journal of Web Semantics, vol. 1, n 4, p. 1-19, 2004.

Carré B., Méthodologie orientée objet pour la représentation des connaissances. Concepts de points de vue, de représentation multiple et évolutive d'objet, Thèse d'informatique, Université des Sciences et Techniques de Lille Flandres Artois, 1989.

Carré B., Geib J.-M., "The Point of View Notion for Multiple Inheritance", Proceedings of Oopsla-ECOOP'90, Ottawa, Canada, ACM SIGPLAN Notices, vol. 25, $n^{\circ}$ 10, p. 312-321, 1990.

d'Aquin M., Bouthier C., Brachais S., Lieber J., Napoli A., "Knowledge Edition and Maintenance Tools for a Semantic Portal in Oncology", International Journal on HumanComputer Studies, vol. 62, n 5, p. 619-638, 2005.

d'Aquin M., Brachais S., Lieber J., Napoli A., "Decision Support and Knowledge Management in Oncology using Hierarchical Classification", Proc. of the Symposium on Computerized Guidelines and Protocols, CGP-2004, vol. 101 of Studies in Health Technology and Informatics, IOS Press, p. 16-30, 2004a.

d'Aquin M., Lieber J., Napoli A., « Représentation de points de vue pour le raisonnement à partir de cas », J. Euzenat, B. Carré (eds), Langages et modèles à objets, Lille (LMO'04), Hermès, L'objet vol. 10, n 2-3, p. 245-258, 2004b.

Davis H. E., VIEWS : Multiple Perspectives and Structured Objects in a Knowledge Representation Language, Bachelor and master of science thesis, MIT, 1987. 
Dekker L., FROME : Représentation multiple et classification d'objets avec points de vue, Thèse d'université, Université des sciences et technologies de Lille, Juin, 1994.

Ducournau R., « La logique des objets », R. Ducournau, J. Euzenat, G. Masini, A. Napoli (eds), Langages et modèles à objets - Etat des recherches et perspectives, Collection Didactique D-019, INRIA, Le Chesnay, p. 351-379, 1998.

Euzenat J., « Représentation de connaissances par objets », R. Ducournau, J. Euzenat, G. Masini, A. Napoli (eds), Langages et modèles à objets - Etat des recherches et perspectives, Collection Didactique D-019, INRIA, Le Chesnay, p. 293-319, 1998.

Fensel D., Hendler J., Lieberman H., Wahlster W. (eds), Spinning the Semantic Web, The MIT Press, Cambridge, Massachusetts, 2003.

Ferber J., Objets et agents : une étude des structures de représentation et de communications en intelligence artificielle, Thèse de doctorat d'état, Université Pierre et Marie Curie - Paris 6, Juin, 1989.

Ferber J., Volle P., "Using Coreference in Object Oriented Representations", Proceedings of the 8th ECAI, Munich, Germany, p. 238-240, 1988.

Ganter B., Wille R., Formal Concept Analysis, Springer, Berlin, 1999.

Ghidini C., Guinchiglia F., "Local models semantics, or contextual reasoning = locality + compatibility", Artificial Intelligence, vol. 127, n 2, p. 221-259, 2001.

Guarino N., "Understanding, Building, and Using Ontologies", International Journal of Human-Computer Studies (IJHCS), vol. 46, n² 2-3, p. 293-310, 1997.

Guinchiglia F., Serafini L., "Multilanguage hierarchical logics, or : how to do without modal logics", Artificial Intelligence, vol. 65, n 1, p. 29-70, 1994.

Hautamäki A., "Points of View and Their Logical Analysis", Acta Philosophica Fennica, 1986.

Mariño O., Raisonnement classificatoire dans une représentation multi-points de vue, Thèse d'université, Université Joseph Fourier - Grenoble 1, Octobre, 1993.

Mariño O., Rechenmann F., Uvietta P., "Multiple Perspectives and Classification Mechanism in Object-Oriented Representation", Proceedings of the 9th ECAI, Stockholm, Sweden, p. 425430, 1990.

Masini G., Napoli A., Colnet D., Léonard D., Tombre K., Les langages à objets, InterEditions, Paris, 1989.

McCarthy J., "Notes on Formalizing Context", Proc. of the International Joint Conference on Artificial Intelligence, IJCAI'93, 1993.

Nassar M., Analyse/conception par points de vue : le profil VUML, Thèse d'informatique, Institut National Polytechnique de Toulouse, 2005.

Nelson S., Powell T., Humphreys B., "The Unified Medical Language System (UMLS) Project”, Encyclopedia of Library and Information Science, Marcel Dekker, p. 369-378, 2002.

Rector A., Nowlan W., "The GALEN project", Computer Methods and Programs in Biomedicine, vol. 45, p. 75-78, 1993.

Réseau de santé Oncolor (RSO), « Référentiel pour le diagnostic et le traitement $\mathrm{du}$ cancer du sein — partie chimiothérapie néo-adjuvante », http ://www.oncolor.org/referentiels/sein/sein_ttt_adj_neo.htm, 2005a.

Réseau de santé Oncolor (RSO), « Référentiel pour le diagnostic et le traitement du cancer du sein - partie chirurgie des tumeurs opérables d'emblée », http ://www.oncolor.org/referentiels/sein/sein_ttt_chir_arb.htm, 2005b. 
Réseau de santé Oncolor (RSO), « Référentiel pour le diagnostic et le traitement $\mathrm{du}$ cancer du sein - partie radiothérapie », http ://www.oncolor.org/referentiels/sein/sein_ttt_rdth_arb.htm, 2005c.

Réseau de santé Oncolor (RSO), « Référentiel pour le diagnostic et le traitement $\mathrm{du}$ cancer du sein - partie traitement médical adjuvant », http ://www.oncolor.org/referentiels/sein/sein_ttt_adj.htm, 2005d.

Réseau de santé Oncolor (RSO), « Site internet du réseau de santé en cancérologie de Lorraine, Oncolor», http ://www.oncolor.org/, 2005e.

Ribière M., Dieng R., "Introduction of Viewpoints in Conceptual Graph Formalism”, Proc. of the 5th International Conference on Conceptual Structures, ICCS'97, 1997.

Schreiber G., Akkermans H., Anjewierden A., de Hoog R., Shadbolt N., van de Velde W., Wielinga B., Knowledge Engineering and Management : the CommonKADS Methodoloy, The MIT Press, Cambridge, MA, 1999.

Serafini L., Tamilin A., "Local Tableaux for Reasoning in Distributed Description Logics", V. Haarslev, R. Moeller (eds), Proc. of the International Workshop on Description Logics, DL'04, p. 100-109, 2004.

Serafini L., Tamilin A., "DRAGO : Distributed reasoning architecture for the semantic web", A. Gomez-Perez, J. Euzenat (eds), Proc. of the Second European Semantic Web Conference (ESWC'05), vol. 3532 of Lecture Notes in Computer Science, Springer, p. 361-376, May, 2005.

Shvaiko P., Euzenat J., "A Survey of Schema-Based Matching Approaches”, Journal on Data Semantics, vol. IV, p. 146-171, 2005.

Staab S., Studer R. (eds), Handbook on Ontologies, Springer, Berlin, 2004.

Stuckenschmidt H., van Harmelen F., Serafini L., Giunchiglia F., "Using C-OWL for the Alignement and Merging of Medical Ontologies", Proc. of the KR workshop on Knowledge representation in medicine, KRMED 2004, 2004.

WOWG, "OWL Web Ontology Language Guide", W3C recommendation (W3C Web Ontology Working Group), 2004. http ://www.w3.org/TR/owl-guide/. 